\title{
Chiral Molecular Ruby $\left[\mathrm{Cr}(\mathrm{dqp})_{2}\right]^{3+}$ with Long-Lived Circularly Polarized
}

\section{Luminescence}

Juan-Ramón Jiménez, Benjamin Doistau, Carlos M. Cruz, Céline Besnard, Juan M. Cuerva, Araceli G. Campaña and Claude Piguet

Supporting Information

(32 pages) 


\section{Appendix 1. Experimental section.}

\section{Solvents and starting materials}

Reagent grade acetonitrile (ACN) was distilled from $\mathrm{CaH}_{2}$. All other chemicals were purchased from commercial suppliers and used without further purification. The ligand 2,6di(quinolin-8-yl)pyridyl (dqp), $\mathrm{Cr}\left(\mathrm{CF}_{3} \mathrm{SO}_{3}\right)_{2} \cdot 2 \mathrm{H}_{2} \mathrm{O}$ and $\mathrm{Na}_{2} \mathrm{Sb}_{2}\left[(+)-\mathrm{C}_{4} \mathrm{O}_{6} \mathrm{H}_{2}\right]_{2} \cdot 5 \mathrm{H}_{2} \mathrm{O}$ were prepared according to the published method. ${ }^{\text {S1-S3 }}$

\section{Synthesis of $\mathrm{rac}-\left[\mathrm{Cr}(\mathrm{dqp})_{2}\right]\left(\mathrm{SO}_{3} \mathrm{CF}_{3}\right)_{3}(1)$}

In a glovebox, $\mathrm{Cr}\left(\mathrm{CF}_{3} \mathrm{SO}_{3}\right)_{2} \cdot 2 \mathrm{H}_{2} \mathrm{O}(44 \mathrm{mg}, 0.450 \mathrm{mmol}, 1$ eq. $)$ and $(n-\mathrm{Bu})_{4} \mathrm{NCF}_{3} \mathrm{SO}_{3}(40 \mathrm{mg}$, $0.450 \mathrm{mmol}, 1$ eq.) were charged into a Schlenk tube. The Schlenk tube was transferred into the lab and degassed acetonitrile $(4 \mathrm{~mL})$ was added. The obtained blue-sky solution was added into a previously prepared solution containing the ligand 2,6-di(quinolin-8-yl)pyridyl (dqp) (80 mg, $0.895 \mathrm{mmol}, 1.6$ eq.) in degassed acetonitrile $(10 \mathrm{~mL})$. The colour of the mixture turned progressively to deep red-brown during the addition. After being stirred at 45 ${ }^{\circ} \mathrm{C}$ for 2 hours, the Schlenk tube was open to air and the colour of the solution slightly changed to red-orange. After one hour stirring at RT, acetonitrile was evaporated and the resulting solid was re-solubilized in acetonitrile $(3 \mathrm{~mL})$ and the mixture filtered. Slow evaporation of the previous solution led to orange plate-like crystals suitable for single crystal diffraction after $12 \mathrm{~h}$. Crystals were filtered, washed with cold $\mathrm{CH}_{3} \mathrm{CN}$ and dried under vacuum (Yield 60\%). ESI-MS $\left(\mathrm{CH}_{3} \mathrm{CN}\right) \mathrm{m} / \mathrm{z}$ : $\left.\left[\mathrm{Cr}(\mathrm{dqp})_{2}\right]\left(\mathrm{SO}_{3} \mathrm{CF}_{3}\right)_{2}\right]^{+}$calc: 1016.1 , found: 1016.3. $\left.\left[\mathrm{Cr}(\mathrm{dqp})_{2}\right]\left(\mathrm{SO}_{3} \mathrm{CF}_{3}\right)\right]^{2+}$ calc: 433.5 , found: 433.6 . $\left.[\mathrm{Cr}(\mathrm{dqp})]\left(\mathrm{SO}_{3} \mathrm{CF}_{3}\right)\right]^{+}$calc: 534.0 , found: 534 (figure S3). Elemental analysis: Calcd for $\mathrm{C}_{49} \mathrm{H}_{30} \mathrm{CrF}_{9} \mathrm{~N}_{6} \mathrm{O}_{9} \mathrm{~S}_{3} \cdot 2 \mathrm{H}_{2} \mathrm{O}: \mathrm{C}, 49.07 ; \mathrm{H}$, $2.83 ; \mathrm{N}, 7.01$. Found C, 49.06; H, 2.82; N, 7.04.

\section{Chiral resolution}

The rac- $\left[\mathrm{Cr}(\mathrm{dqp})_{2}\right]^{3+}(18 \mathrm{mg})$ was absorbed onto a $90 \mathrm{~cm}$ length chromatography column charged with Sephadex SP-C25 ion-exchange resin. The complex was eluted with an aqueous solution of $\mathrm{Na}_{2} \mathrm{Sb}_{2}\left[(+)-\mathrm{C}_{4} \mathrm{O}_{6} \mathrm{H}_{2}\right]_{2}, 5 \mathrm{H}_{2} \mathrm{O}(0.15 \mathrm{M})$ and two successive orange bands can be separated (fraction I and II successively). Few drops of an aqueous saturated $\mathrm{KPF}_{6}$ solution were added to the previous fractions resulting in a yellow precipitate. The solid was separated by centrifugation (15 $\mathrm{min}$ at $80000 \mathrm{rpm})$ and washed twice with water. The solid was dissolved in acetone and filtered to remove the excess of tartrate salt. Evaporation of the solvent results in a yellow solid which was dried under vacuum leading to $P P-\left[\mathrm{Cr}(\mathrm{dqp})_{2}\right]^{3+}(7$ $\mathrm{mg}$, from fraction I) and $\left.M M-\left[\mathrm{Cr}(\mathrm{dqp})_{2}\right]^{3+}(8 \mathrm{mg} \text {, from fraction II). FTIR: rac-[Cr(dqp })_{2}\right]^{3+} v$ $\left(\mathrm{cm}^{-1}\right)$ : 3111(w), 3082(w), 1610(m), 1575(m), 1568(m), 1512(m), 1454(m), 1384(m), 1367(m), 1313(m), 1284(m), 1242(m), 1218(m), 1145(m), 1028(m), 829(s, P-F), 765(s, P-F), 
649(m), 555(s), 470(m), 401(m), 331(m); PP-[Cr(dqp) $)_{2}\left(\mathrm{PF}_{6}\right)_{3} \cdot \mathrm{C}_{3} \mathrm{H}_{6} \mathrm{O} \quad v\left(\mathrm{~cm}^{-1}\right): 3124(\mathrm{w})$, 3086(w), 2922(w), 2850(w), 1664(m, C=O), 1608(m), 1577(m), 1573(m), 1512(m), 1454(m), 1382(m), 1367(m), 1313(m), 1280(m), 1245(m), 1213(m), 1145(m), 1132(m), 812(s, P-F), 794(s, P-F), 649(m), 553(s), 472(m), 401(m), 333(m); MM-[Cr(dqp) $\left.)_{2}\right]^{3+} \cdot \mathrm{C}_{3} \mathrm{H}_{6} \mathrm{O} \quad v\left(\mathrm{~cm}^{-1}\right)$ : 3111(w), 3070(w), 2922(w), 2852(w), 1706(m, C=O), 1608(m), 1587(m), 1571(m), 1512(m), 1454(m), 1367(m), 1365(m), 13099(m), 1280(m), 1244(m), 1228(m), 1147(m), 1029(m), 813(s, P-F), 796(s, P-F), 649(m), 555(s), 470(m), 401(m), 331(m).

\section{Spectroscopic and analytical measurements.}

Pneumatically-assisted electrospray (ESI) mass spectrum was recorded from $10^{-4} \mathrm{M}$ solution on an Applied Biosystems API 150EX LC/MS System equipped with a Turbo Ionspray source $^{\circledR}$. Elemental analyses were performed by K. L. Buchwalder from the Microchemical Laboratory of the University of Geneva. FT-IR spectra were collected in the $200-4000 \mathrm{~cm}^{-1}$ range. Measurements were carried out on a Bruker Tensor 27 FT-IR instrument using the attenuated total reflection (ATR) technique on solid samples (with a $4 \mathrm{~cm}^{-1}$ resolution). The intensity of the absorption band is indicated as w (weak), m (medium), s (strong). Absorption spectra in water solution were recorded using a Lambda 1050 Perkin Elmer spectrometer (quartz cell path length $1 \mathrm{~cm}$ or $1 \mathrm{~mm}, 290-800 \mathrm{~nm}$ domain, $2 \times 10^{-4} \mathrm{~mol} / \mathrm{L}$ and $650-800 \mathrm{~nm}$ domain, $7.7 \mathrm{mM}$ ). Emission spectra (excitation at $355 \mathrm{~nm}$ ) and excitation spectra were recorded from either room temperature or frozen solution samples (for frozen solutions: $\mathrm{H}_{2} \mathrm{O} / \mathrm{DMSO} 5 / 5$ at $\left.\mathrm{C} \approx 5 \times 10^{-3} \mathrm{~mol} / \mathrm{L}\right)$, or freeze-pump-thaw degassed water solution $\left(\mathrm{C} \approx 10^{-4}\right.$ $\mathrm{mol} / \mathrm{L}$ ) for room temperature solutions $293 \mathrm{~K}$, with a Fluorolog (Horiba Jobin-Yvon), equipped with iHR320, a Xenon lamp 450 Watt Illuminator (FL-1039A/40A) and a watercooled photo multiplier tube (PMT Hamamatsu R2658 or R928), and corrected for the spectral response of the system. For time-resolved experiments, the decay curves were recorded from previously excited samples at $77 \mathrm{~K}$ and $293 \mathrm{~K}$, with a photomultiplier (Hamamatsu R2658 or R928) and a digital oscilloscope (Tektronix MDO4104C). Pulsed excitation at $355 \mathrm{~nm}$ was obtained with the third harmonic of a pulsed Nd:YAG laser (Quantel Qsmart 850). Low temperature (77 K) was achieved using liquid quartz transparent Deward filled with liquid $\mathrm{N}_{2}$ in the centre of which samples were placed. Samples solutions $\left(\mathrm{H}_{2} \mathrm{O} / \mathrm{DMSO} 5 / 5\right.$ at $\left.\mathrm{C} \approx 5 \times 10^{-3} \mathrm{~mol} / \mathrm{L}\right)$, were introduced in quartz tube $(4 \mathrm{~mm}$ interior diameter) and introduced in sample holder of the Dewar. The oxygen free decay curve measurements were done at $293 \mathrm{~K}$ on water solutions of the complex $\left(\mathrm{C} \approx 10^{-4} \mathrm{~mol} / \mathrm{L}\right)$. The complex in a quartz tube was dissolved in water then degassed by freeze-pump-thaw, and finally filled with argon. Aerated solutions were prepared by using no-degassed water as 
solvent. All emission quantum yields were measured according to an absolute method which makes use of an integration sphere. The sample was put into a 2-3 mm quartz capillary. The measurement was carried out by following the previously reported method (IUPAC Techinical Report). ${ }^{\mathrm{S} 4}$ The measurements were achieved in $\mathrm{H}_{2} \mathrm{O}$, at room temperature and $\lambda_{\text {exc }}$ $=410 \mathrm{~nm}$ (manifold $\mathrm{Cr}\left({ }^{4} \mathrm{~T}_{2} \leftarrow{ }^{4} \mathrm{~A}_{2}\right) / \mathrm{LMCT}$ ). Molar concentration were adjusted to $1 \times 10^{-5} \mathrm{M}$. Electronic circular dichroism (ECD) spectra were recorded on a Jasco J-815 spectropolarimeter at $20^{\circ} \mathrm{C}$ in a $1 \mathrm{~cm}$ cuvette. The ECD spectra showed in this work are an average spectra calculated after 3 scans (each one). For ECD measurements a fixed $0.1 \mathrm{~s}$ of integration time was selected. The Circularly Polarized Luminescence (CPL) were recorded in an Olis DSM172 spectrophotometer equipped with a xenon lamp of $150 \mathrm{~W}$. The spectra were recorded at $5 \times 10^{-5} \mathrm{M}$ concentrations in HPLC grade solvents and room temperature. A fixed wavelength of $390 \mathrm{~nm}$ provided by a LED source was used, $1.0 \mathrm{~s}$ of integration time was selected, the CPL spectra showed in this work are an average spectra calculated after 10 scans (each one). Optical rotation was recorded on a Perkin-Elmer 341 polarimeter at $2 \times 10^{-4} \mathrm{M}$ in HPLC grade acetonitrile, using a $10 \mathrm{~cm}$ path cell at room temperature. Deaerated solution were prepared from degassed acetonitrile into a glovebox. Aerated solutions were prepared by using no-degassed acetonitrile as solvent.

\section{Time-dependent DFT calculations.}

TD-DFT calculations were performed based on the obtained XRD geometry with the Gaussian 09 package $^{\mathrm{S} 5}$ by means of the exchange-correlation B3LYP functional. The LANL2DZ basis set containing the effective core potential (ECP) were applied for Cr. The 6$31+\mathrm{G}$ basis set was used for $\mathrm{H}, \mathrm{C}$ and $\mathrm{N}$ atoms. The solvent effect was induced in acetonitrile by means of the polarizable continuum model (PCM). Thirty vertical transitions were calculated. Gaussian band shape with a bandwidth of $0.12 \mathrm{eV}$ was used to simulate the UVvis and ECD spectra.

\section{X-Ray Crystallography.}

Summary of crystal data, intensity measurements and structure refinements for rac$\left[\mathrm{Cr}(\mathrm{dqp})_{2}\right]^{3+}$ and $M M-\left[\mathrm{Cr}(\mathrm{dqp})_{2}\right]^{3+}$ compound were collected in Tables S1 and S4 (ESI). The crystals were mounted on MiTeGen cryoloops with protection oil. X-ray data collections were performed with an Agilent SuperNova Dual diffractometer equipped with a CCD Atlas detector $(\mathrm{Cu}[\mathrm{K \alpha}]$ radiation) or a Rigaku symergy $\mathrm{S}$ equipped with an hypix detector (we thank Fraser White and the Rigaku team for assistance during data collection). The structures were solved in SHELXT by using dual space methods. ${ }^{\mathrm{S} 6}$ Full-matrix least-square refinements on $F^{2}$ 
were performed with SHELXLS7 within the Olex2 program. ${ }^{\mathrm{S} 8}$ CCDC 1920463-1920464 contain the supplementary crystallographic data. The cif files can be obtained free of charge on www.ccdc.cam.ac.uk. 


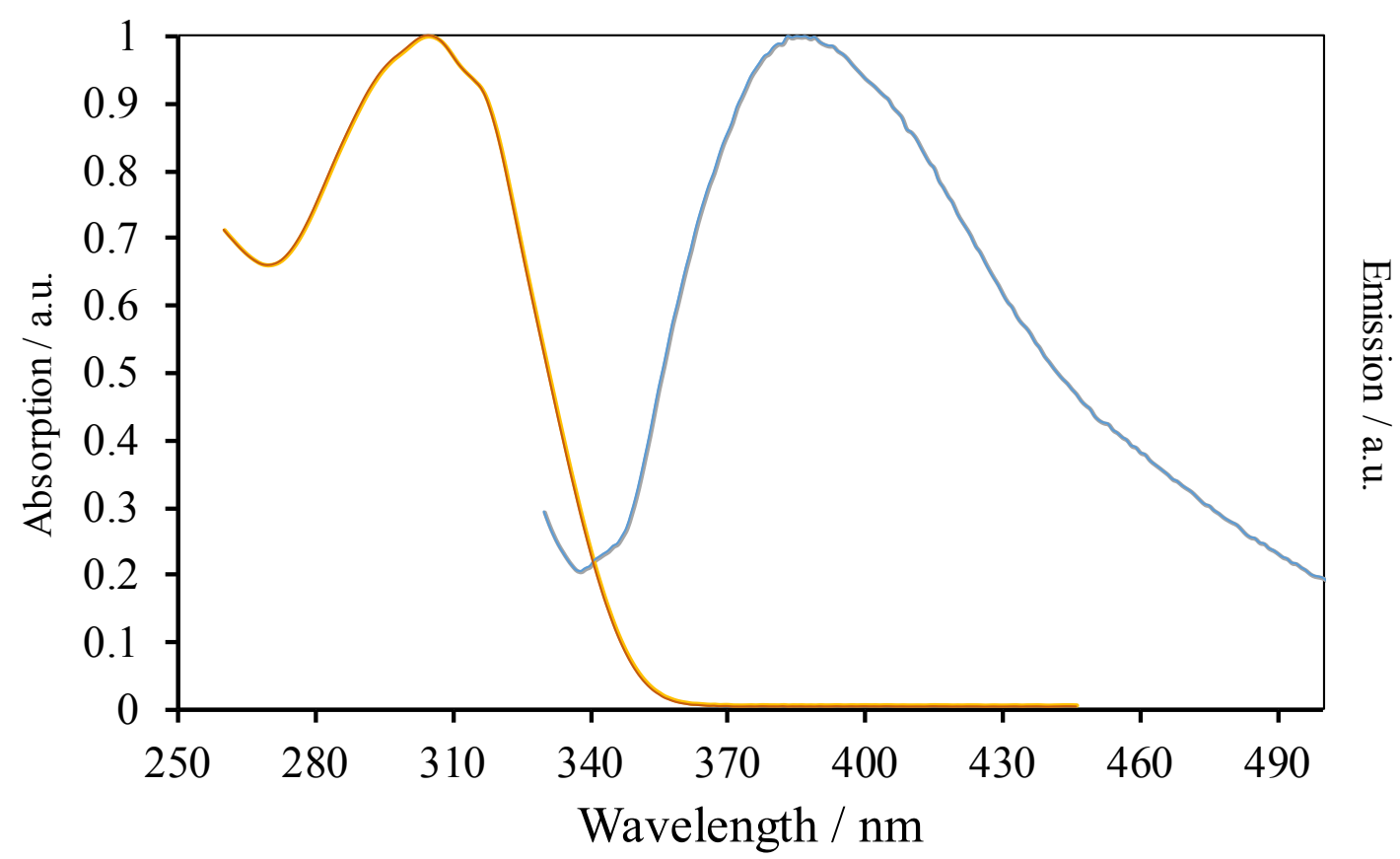

Figure S1. Normalized absorption spectrum (orange) and emission spectrum (blue) of the ligand 2,6-di(quinolin-8-yl)pyridyl (dqp). $\left(\mathrm{CH}_{3} \mathrm{CN}, c=10^{-4} \mathrm{M}\right) . \lambda_{\mathrm{exc}}=320 \mathrm{~nm}$. 


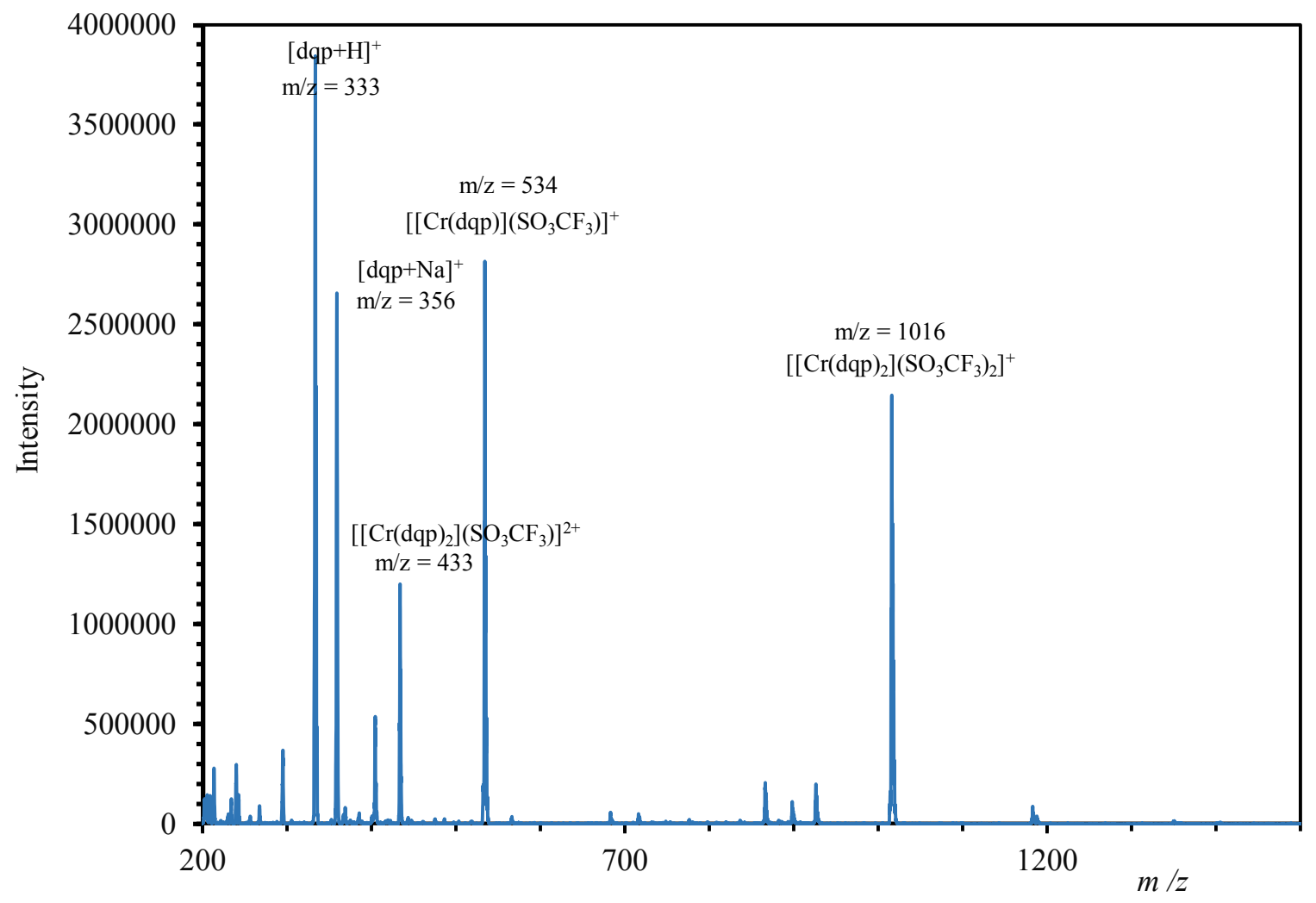

Figure S2. ESI-MS of $\left[\mathrm{Cr}(\mathrm{dqp})_{2}\right]\left(\mathrm{SO}_{3} \mathrm{CF}_{3}\right)_{3}$ in positive mode (solvent $\mathrm{CH}_{3} \mathrm{CN}, 10^{-4} \mathrm{M}$ ). 


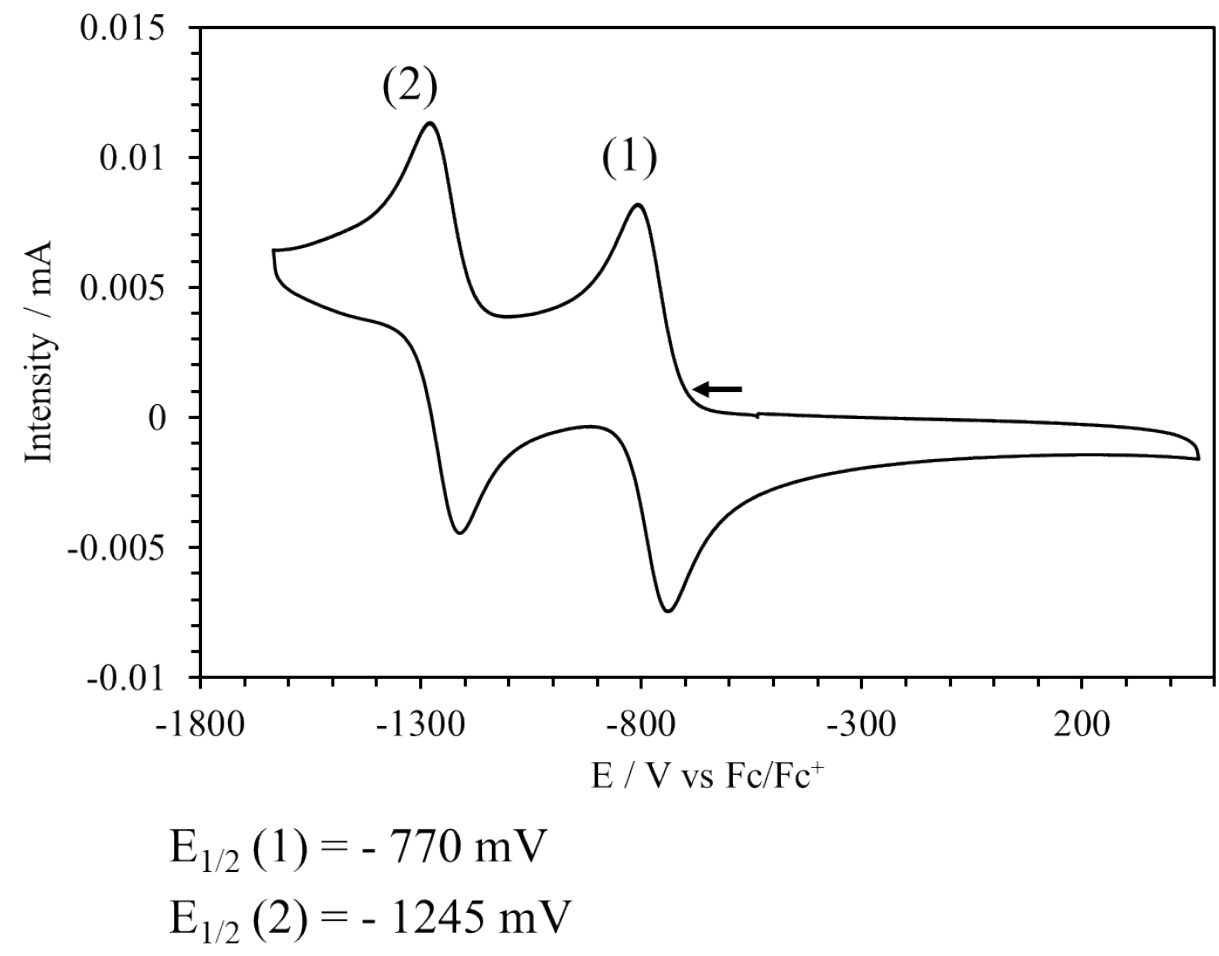

Figure S3. Cyclic voltammogram of $\left[\mathrm{Cr}(\mathrm{dqp})_{2}\right]^{3+}$ in $\mathrm{CH}_{3} \mathrm{CN}+0.1 \mathrm{M} \mathrm{NBu}_{4} \mathrm{PF}_{6}\left(c=10^{-3} \mathrm{M}\right)$. Working electrode: mercury; counter Electrode: silver; Reference Electrode: Silver Chloride. Scan rate $100 \mathrm{mV} \cdot \mathrm{s}^{-1}$. 
Table S1. Crystal data and structure refinement for $r a c$ - $\left[\mathrm{Cr}(\mathrm{dqp})_{2}\right]\left(\mathrm{SO}_{3} \mathrm{CF}_{3}\right)_{3}(\mathbf{1})$

$\begin{array}{ll}\text { Empirical formula } & \mathrm{C}_{51} \mathrm{H}_{33} \mathrm{CrF}_{9} \mathrm{~N}_{7} \mathrm{O}_{9} \mathrm{~S}_{3} \\ \text { Formula weight } & 1207.02 \\ \text { Temperature/K } & 180.15 \\ \text { Crystal system } & \text { triclinic } \\ \text { Space group } & \mathrm{P}-1 \\ a / \AA & 11.1216(4) \\ b / \AA & 13.1420(4) \\ c / \AA & 17.3331(7) \\ \alpha /{ }^{\circ} & 86.510(3) \\ \beta /{ }^{\circ} & 80.088(3) \\ \gamma /{ }^{\circ} & 84.768(3) \\ \text { Volume/ } \AA^{3} & 2482.55(15) \\ Z & 2 \\ \rho_{\text {calc }} \text { g/cm } & \\ \mu / \text { mm- } 1 & 1.615 \\ F(000) & 3.967 \\ \text { Frystal size/mm } & 1251=0.043 \\ \text { Radiation } & \end{array}$


Table S2. Selected bond distances $(\AA)$, bond angles $\left(^{\circ}\right)$ in $r a c-\left[\mathrm{Cr}(\mathrm{dqp})_{2}\right]\left(\mathrm{SO}_{3} \mathrm{CF}_{3}\right)_{3}(\mathbf{1})$.

\begin{tabular}{ccc}
\hline Atom & Atom & Length $/ \AA$ \\
\hline Cr1 & N6 & $2.0660(14)$ \\
Cr1 & N5 & $2.0506(14)$ \\
Cr1 & N4 & $2.0687(14)$ \\
Cr1 & N3 & $2.0650(13)$ \\
Cr1 & N2 & $2.0461(14)$ \\
Cr1 & N1 & $2.0619(13)$ \\
\hline
\end{tabular}

\begin{tabular}{|c|c|c|c|}
\hline Atom & Atom & Atom & Angle $/^{\circ}$ \\
\hline N6 & $\mathrm{Cr} 1$ & N4 & $174.97(6)$ \\
\hline N5 & $\mathrm{Cr} 1$ & N6 & $87.52(6)$ \\
\hline N5 & $\mathrm{Cr} 1$ & N4 & $87.45(6)$ \\
\hline N5 & Cr1 & N3 & $92.73(5)$ \\
\hline N5 & $\mathrm{Cr} 1$ & N1 & $92.67(5)$ \\
\hline N3 & $\mathrm{Cr} 1$ & N6 & $88.17(6)$ \\
\hline N3 & $\mathrm{Cr} 1$ & N4 & $91.90(5)$ \\
\hline N2 & $\mathrm{Cr} 1$ & N6 & $92.55(6)$ \\
\hline N2 & $\mathrm{Cr} 1$ & N5 & $179.83(6)$ \\
\hline N2 & $\mathrm{Cr} 1$ & N4 & $92.48(6)$ \\
\hline N2 & $\mathrm{Cr} 1$ & N3 & $87.12(5)$ \\
\hline N2 & $\mathrm{Cr} 1$ & N1 & $87.48(5)$ \\
\hline N1 & $\mathrm{Cr} 1$ & N6 & $92.38(5)$ \\
\hline N1 & $\mathrm{Cr} 1$ & N4 & $88.03(5)$ \\
\hline N1 & $\mathrm{Cr} 1$ & N3 & $174.59(5)$ \\
\hline
\end{tabular}

Table S3. Interplanar angles $\left(^{\circ}\right)$ in $r a c-\left[\mathrm{Cr}(\mathrm{dqp})_{2}\right]\left(\mathrm{SO}_{3} \mathrm{CF}_{3}\right)_{3}(\mathbf{1})$. 
$\mathrm{N} 1 \mathrm{C} 1 \mathrm{C} 2 \mathrm{C} 3 \mathrm{C} 4 \mathrm{C} 5 \mathrm{C} 6 \mathrm{C} 7 \mathrm{C} 8 \mathrm{C} 9$

$\mathrm{N} 2 \mathrm{C} 10 \mathrm{C} 11 \mathrm{C} 12 \mathrm{C} 13 \mathrm{C} 14$

N3 C15 C16 C17 C18 C19 C20 C21 C22 C23

N4 C24 C25 C26 C27 C28 C29 C30 C31 C32

N5 C33 C34 C35 C36 C37

Py5

N6 C38 C39 C40 C41 C42 C43 C44 C45 C46

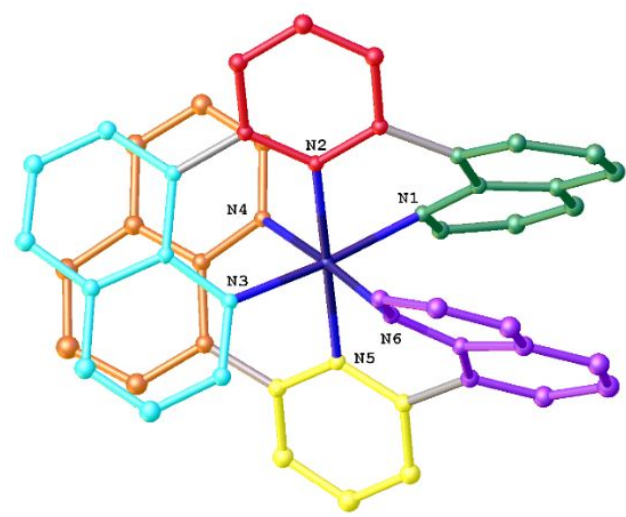

\begin{tabular}{|l|c|c|}
\hline & & RMSD $(\AA)$ \\
\hline P1 & N1 C1 C2 C3 C4 C5 C6 C7 C8 C9 & 0.095 \\
P2 & N2 C10 C11 C12 C13 C14 & 0.007 \\
P3 & N3 C15 C16 C17 C18 C19 C20 C21 C22 C23 & 0.081 \\
P4 & N4 C24 C25 C26 C27 C28 C29 C30 C31 C32 & 0.081 \\
P5 & N5 C33 C34 C35 C36 C37 & 0.011 \\
P6 & N6 C38 C39 C40 C41 C42 C43 C44 C45 C46 & 0.104 \\
\hline
\end{tabular}

RMSD: Root mean square deviation between the atoms and the plane

\begin{tabular}{|l|l|l|l|l|l|l|}
\hline & 1 & 2 & 3 & 4 & 5 & 6 \\
\hline 1 & & 44.6 & 80.2 & 98.6 & 48.2 & 15.3 \\
2 & & & 36.01 & 141.8 & 17.9 & 46.2 \\
3 & & & & 162.16 & 41.9 & 81.8 \\
4 & & & & 144.9 & 101.7 \\
6 & & & & & & 43.2 \\
\hline
\end{tabular}

The error is typically $\pm 0.1^{\circ}$. 


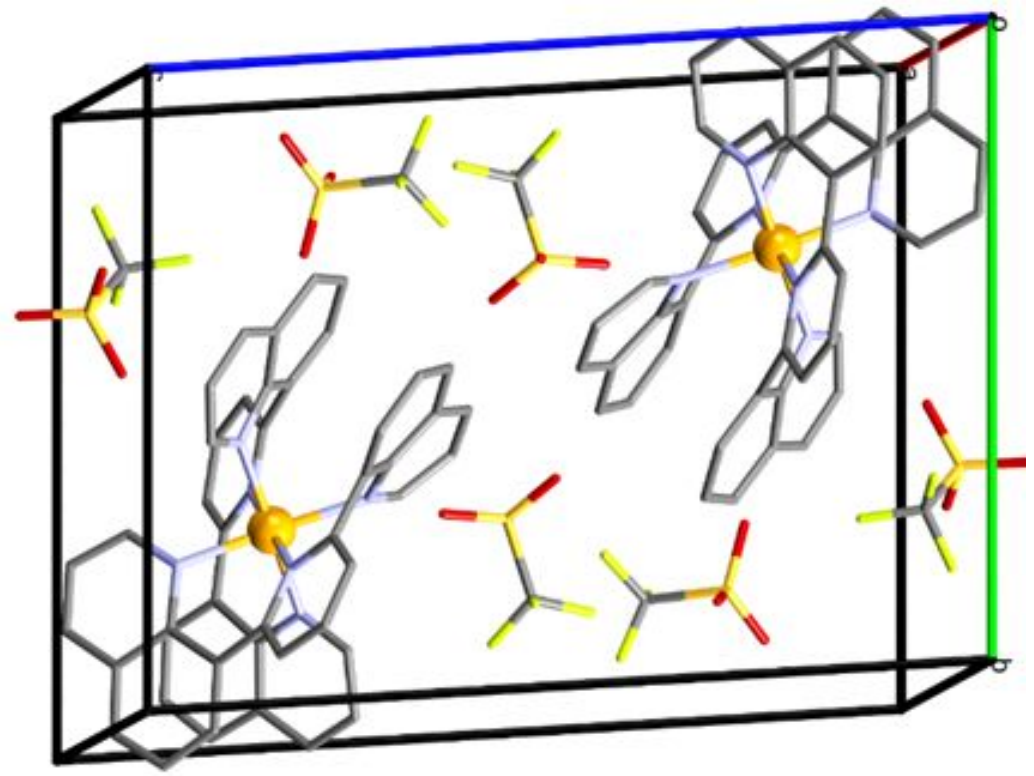

Figure S4. Unit cell for $r a c-\left[\mathrm{Cr}(\mathrm{dqp})_{2}\right]\left(\mathrm{SO}_{3} \mathrm{CF}_{3}\right)_{3}(\mathbf{1})$. Colour code for the atoms: O (red), $\mathrm{S}$ (yellow), C (gray), F (green), N (blue) and Cr (orange)
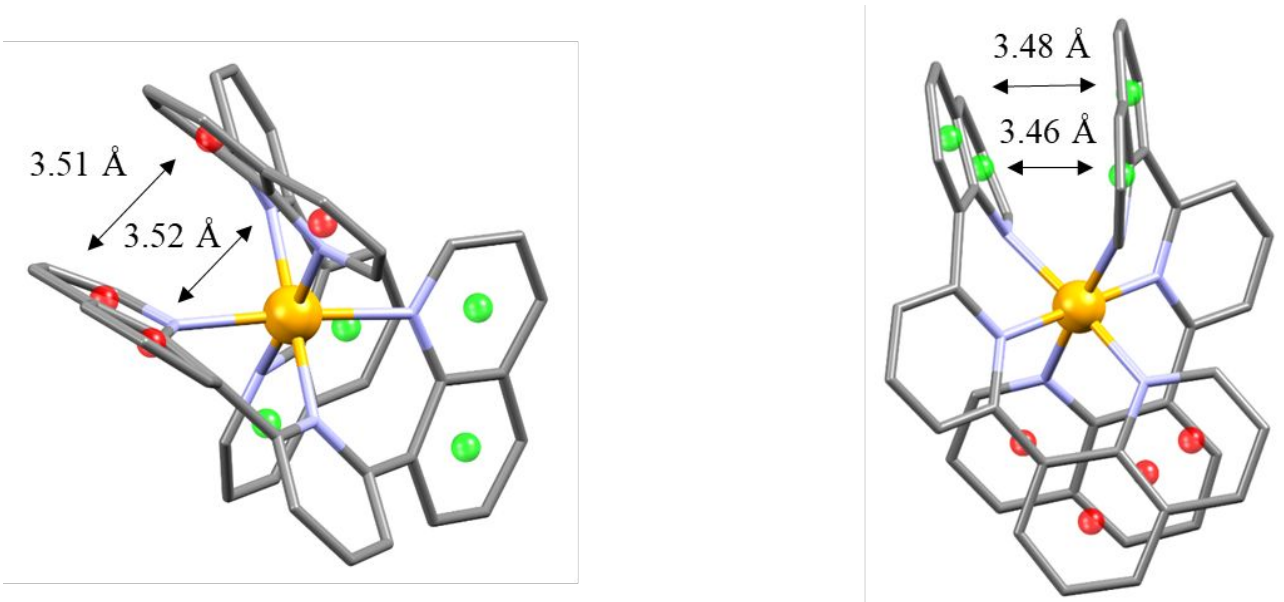

Figure S5. Intramolecular interstrand stacking through $\pi$ interaction involving quinoline rings in rac- $\left[\mathrm{Cr}(\mathrm{dqp})_{2}\right]\left(\mathrm{SO}_{3} \mathrm{CF}_{3}\right)_{3}(\mathbf{1})$. Colour code for the atoms: $\mathrm{C}$ (gray), $\mathrm{F}$ (green), $\mathrm{N}$ (blue) and Cr (orange) 
Table S4. Crystal data and structure refinement for $M M-(-)\left[\mathrm{Cr}(\mathrm{dqp})_{2}\right]\left(\mathrm{PF}_{6}\right)_{3}$.

Empirical formula

Formula weight

Temperature/K

Crystal system

Space group

$a / \AA$

$b / \AA$

$c / \AA$

$\alpha /^{\circ}$

$\beta /{ }^{\circ}$

$\gamma /{ }^{\circ}$

Volume $/ \AA^{3}$

Z

$\rho_{\text {calc }} \mathrm{g} / \mathrm{cm} 3$

$\mu / \mathrm{mm}-1$

$F(000)$

Crystal size $/ \mathrm{mm}^{3}$

Radiation

$2 \Theta$ range for data collection ${ }^{\circ}$

Index ranges

Reflections collected

Independent reflections

Data/restraints/parameters

Goodness-of-fit on $F^{2}$

Final $R$ indexes $[I>=2 \sigma(I)]$

Final $R$ indexes [all data]

Largest diff. peak/hole / e Å-3

Flack parameter
$\mathrm{C}_{52} \mathrm{H}_{44} \mathrm{CrF}_{18} \mathrm{~N}_{6} \mathrm{O}_{3} \mathrm{P}_{3}$

1287.84

$180.00(10)$

orthorhombic

P212121

13.45292(10)

$17.12816(13)$

$23.4250(2)$

90

90

90

$5397.68(7)$

4

1.585

3.598

2612.0

$0.574 \times 0.424 \times 0.24$

$\mathrm{CuK} \alpha(\lambda=1.54184)$

7.548 to 137.692

$-16 \leq h \leq 14,-20 \leq k \leq 20,-27 \leq l \leq 28$

42188

$9897\left[R_{\text {int }}=0.0285, R_{\text {sigma }}=0.0196\right]$

9897/82/822

1.028

$R 1=0.0356, \mathrm{w} R 2=0.0915$

$R 1=0.0363, \mathrm{w} R 2=0.0924$

$0.61 /-0.55$

$0.005(5)$ 
Table S5. Interplanar angles $\left(^{\circ}\right)$ in $M M-(-)\left[\mathrm{Cr}(\mathrm{dqp})_{2}\right]\left(\mathrm{PF}_{6}\right)_{3}$.

Py1 N1 C1 C2 C3 C4 C5 C6 C7 C8 C9

Py2 N2 C10 C11 C12 C13 C14

Py3 N3 C15 C16 C17 C18 C19 C20 C21 C22 C23

Py4 N4 C24 C25 C26 C27 C28 C29 C30 C31 C32

Py5 N5 C33 C34 C35 C36 C37

Py6 N6 C38 C39 C40 C41 C42 C43 C44 C45 C46

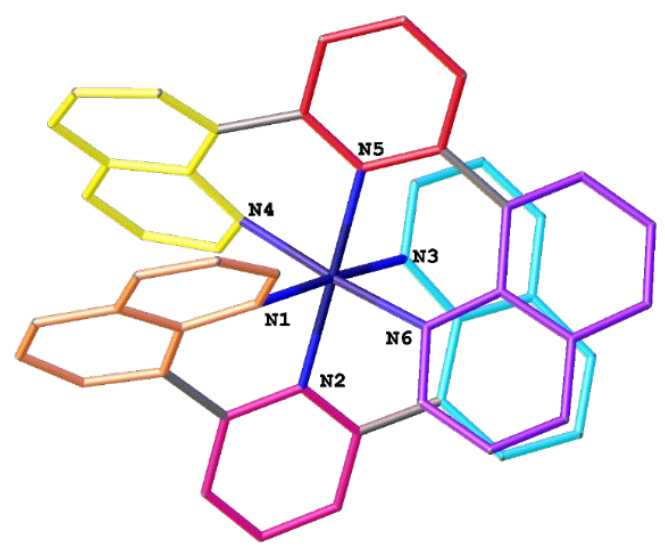

\begin{tabular}{|l|l|l|}
\hline & & RMSD $(\AA)$ \\
\hline P1 & N1 C1 C2 C3 C5 C4 C6 C7 C8 C9 & 0.084 \\
\hline P2 & N2 C10 C11 C12 C13 C14 & 0.010 \\
\hline P3 & N3 C23 C22 C21 C19 C18 C17 C16 C15 C20 & 0.083 \\
\hline P4 & N4 C31 C32 C33 C34 C36 C37 C38 C39 C35 & 0.084 \\
\hline P5 & N5 C40 C41 C42 C43 C44 & 0.005 \\
\hline P6 & N6 C50 C45 C46 C47 C48 C49 C51 C52 C53 & 0.085 \\
\hline
\end{tabular}

RMSD: Root mean square deviation between the atoms and the plane

Angles $\left({ }^{\circ}\right)$.

\begin{tabular}{|l|l|l|l|l|l|l|}
\hline & 1 & 2 & 3 & 4 & 5 & 6 \\
\hline 1 & & 140.6 & 104.0 & 160.9 & 137.8 & 99.7 \\
\hline 2 & & & 37.6 & 45.4 & 20.7 & 43.1 \\
\hline 3 & & & & 81.3 & 45.2 & 19.3 \\
\hline 4 & & & & & 38 & 78.1 \\
\hline 5 & & & & & & 40.2 \\
\hline 6 & & & & & & \\
\hline
\end{tabular}


Table S6. Selected bond distances $(\AA)$, bond angles $\left(^{\circ}\right)$ in $M M-(-)\left[\mathrm{Cr}(\mathrm{dqp})_{2}\right]\left(\mathrm{PF}_{6}\right)_{3}$.

\begin{tabular}{ccc}
\hline Atom & Atom & Length $/ \AA$ \\
\hline Cr1 & N6 & $2.068(2)$ \\
Cr1 & N5 & $2.037(3)$ \\
Cr1 & N4 & $2.063(3)$ \\
Cr1 & N3 & $2.061(3)$ \\
Cr1 & N2 & $2.040(3)$ \\
Cr1 & N1 & $2.070(2)$ \\
\hline
\end{tabular}

\begin{tabular}{|c|c|c|c|}
\hline Atom & Atom & Atom & Angle $/^{\circ}$ \\
\hline N6 & $\mathrm{Cr} 1$ & N4 & $175.77(10)$ \\
\hline N5 & $\mathrm{Cr} 1$ & N6 & $88.14(10)$ \\
\hline N5 & $\mathrm{Cr} 1$ & N4 & $87.65(10)$ \\
\hline N5 & $\mathrm{Cr} 1$ & N3 & $91.90(10)$ \\
\hline N5 & $\mathrm{Cr} 1$ & N1 & $91.45(10)$ \\
\hline N3 & $\mathrm{Cr} 1$ & N6 & $92.82(10)$ \\
\hline N3 & $\mathrm{Cr} 1$ & N4 & $86.89(10)$ \\
\hline N2 & $\mathrm{Cr} 1$ & N6 & $92.05(10)$ \\
\hline N2 & $\mathrm{Cr} 1$ & N5 & 179.61(11) \\
\hline N2 & $\mathrm{Cr} 1$ & N4 & $92.16(10)$ \\
\hline N2 & $\mathrm{Cr} 1$ & N3 & $88.44(10)$ \\
\hline N2 & $\mathrm{Cr} 1$ & N1 & $88.22(10)$ \\
\hline N1 & $\mathrm{Cr} 1$ & N6 & $87.37(10)$ \\
\hline N1 & $\mathrm{Cr} 1$ & N4 & $93.16(10)$ \\
\hline N1 & $\mathrm{Cr} 1$ & N3 & $176.65(10)$ \\
\hline
\end{tabular}




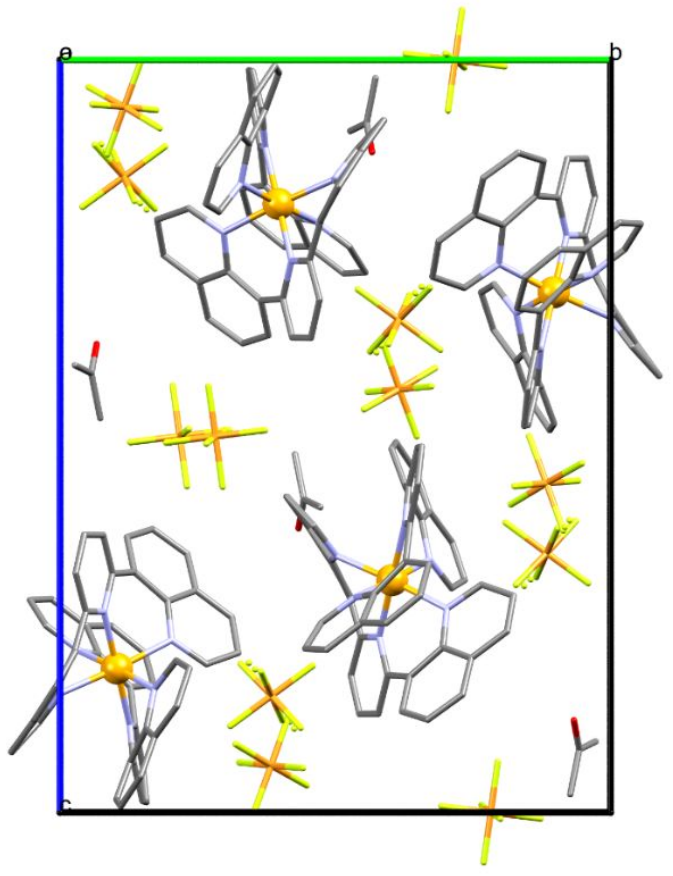

Figure S6. Unit cell for $M M-(-)-\left[\mathrm{Cr}(\mathrm{dqp})_{2}\right]\left(\mathrm{PF}_{6}\right)_{3}$. Colour code for the atoms: $\mathrm{O}$ (red), $\mathrm{S}$ (yellow), C (gray), F (green), N (blue) and Cr (orange).

\section{Left-handed helix}
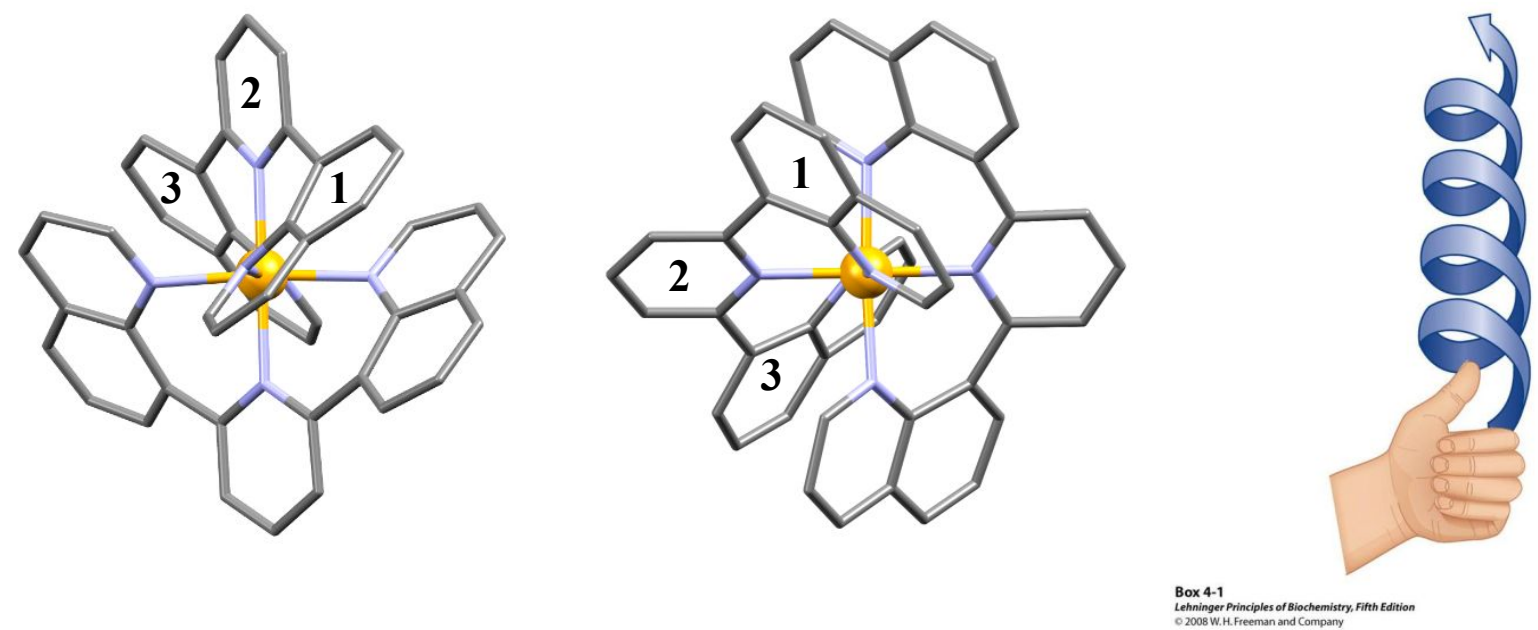

Figure S7. Molecular views of the left-handed helices $(M)$ found in the crystal structure of fraction II. The chiral axis is viewed end-on with one "near" (1) and one "far" (3) quinolines on the axial unit. The nearest quinoline (1) has higher priority than the farest one (3). 


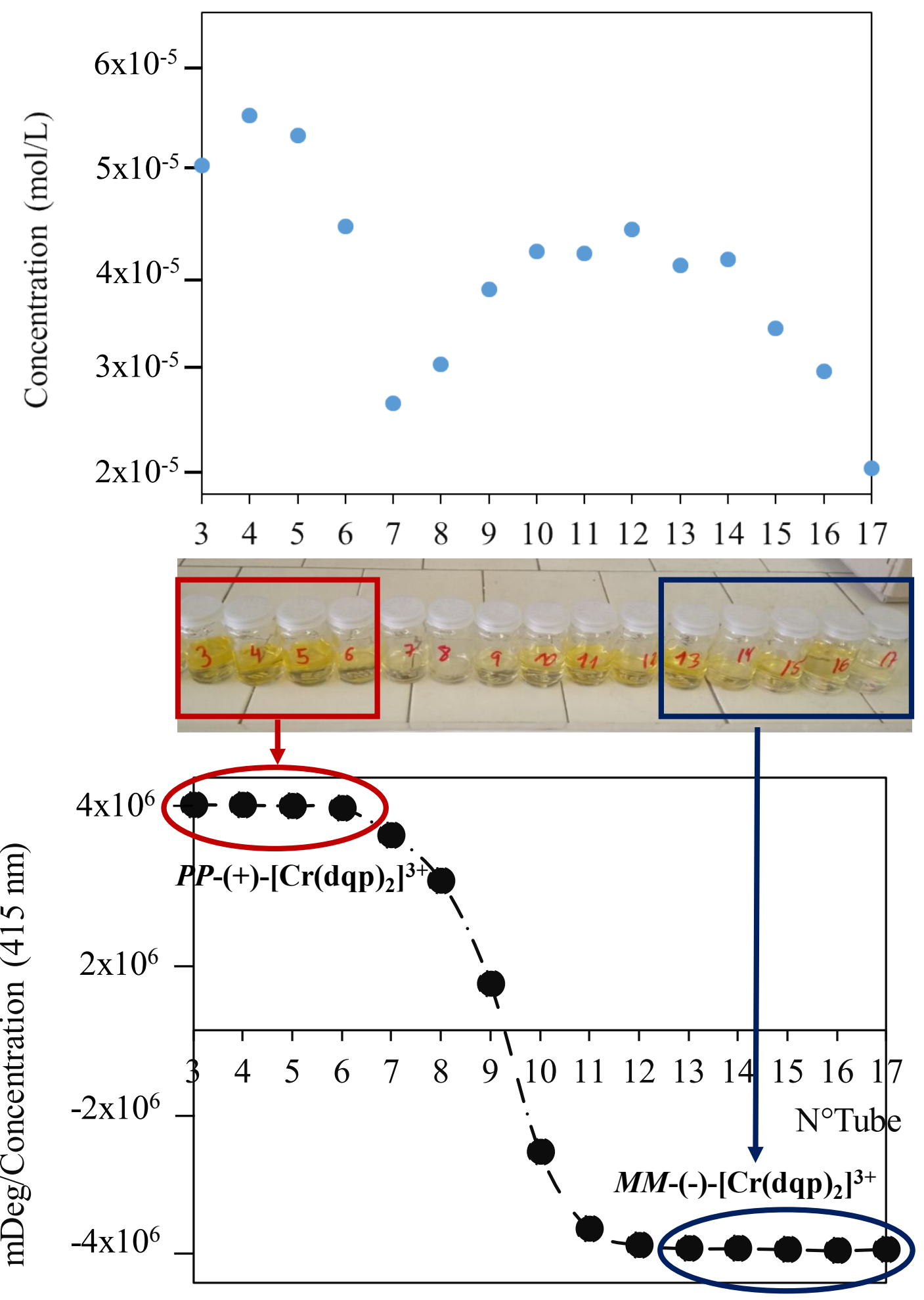

Figure S8. Different fractions obtained from the exchange-cation column. Separation profile obtained by plotting the $\mathrm{mDeg} / \mathrm{concentration}$ (at the maximum $415 \mathrm{~nm}$ ) extracted from the CD spectra of each fraction versus the labelled fraction (bottom). Concentration profile obtained from the absorption spectrum recorded simultaneously for each fraction (top). 

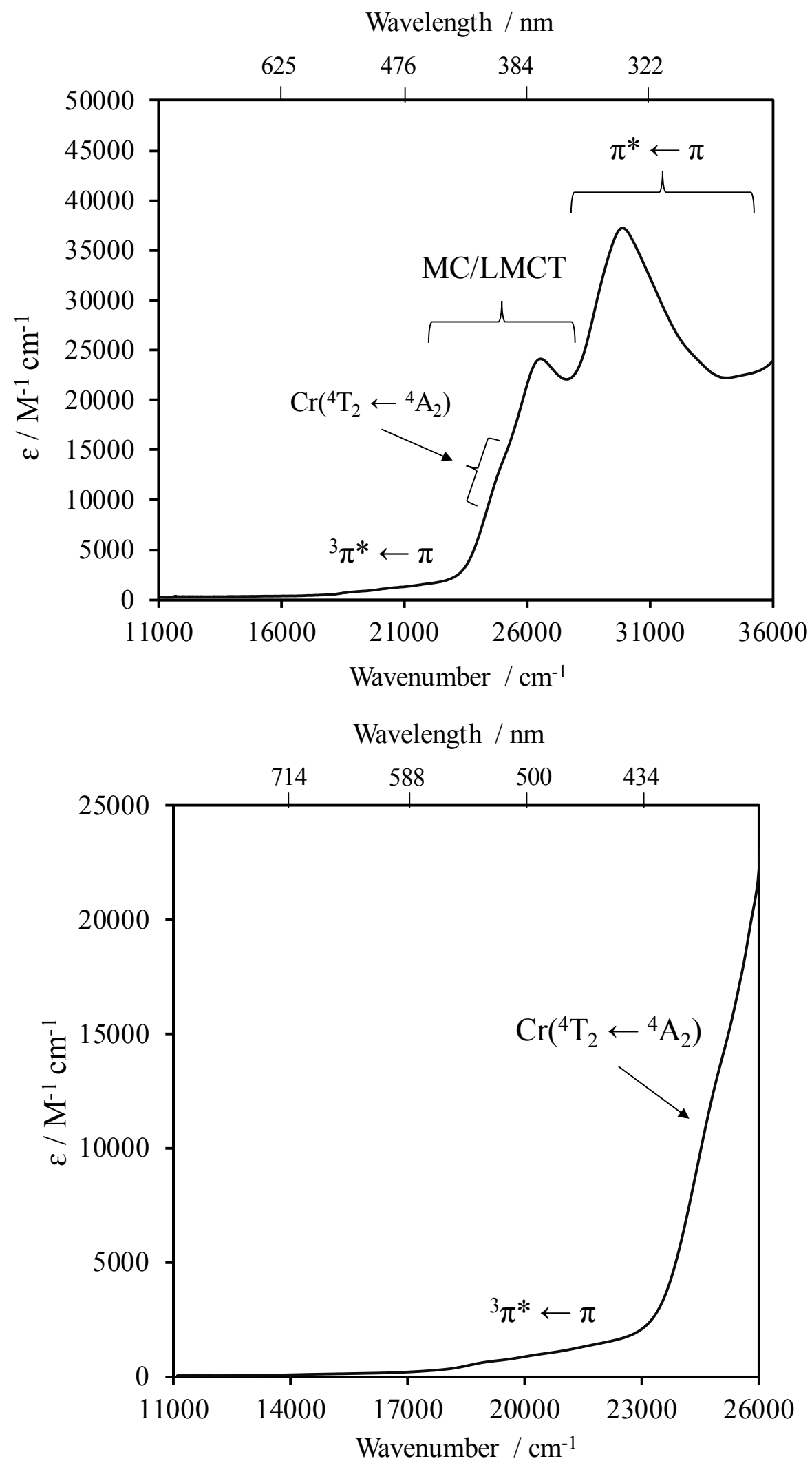

Figure S9. UV-Vis part of the absorption spectrum of the complex $\mathrm{rac}-\left[\mathrm{Cr}(\mathrm{dqp})_{2}\right]^{3+}(\mathbf{1})$ recorded in water at $293 \mathrm{~K}\left(c=10^{-4} \mathrm{M}\right)$ 


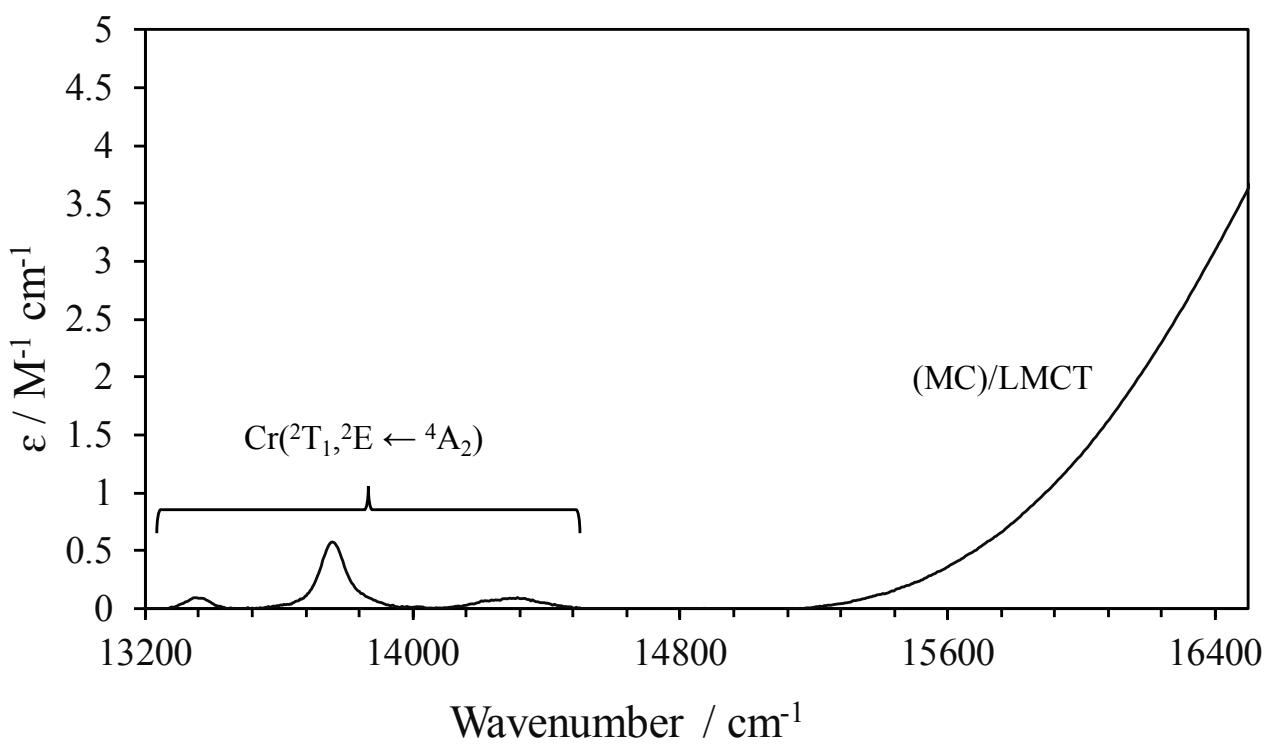

Figure S10. Visible-NIR part of the absorption spectrum of the complex $r a c-\left[\mathrm{Cr}(\mathrm{dqp})_{2}\right]^{3+}$ recorded in water at $293 \mathrm{~K}(C=7.7 \mathrm{mM})$

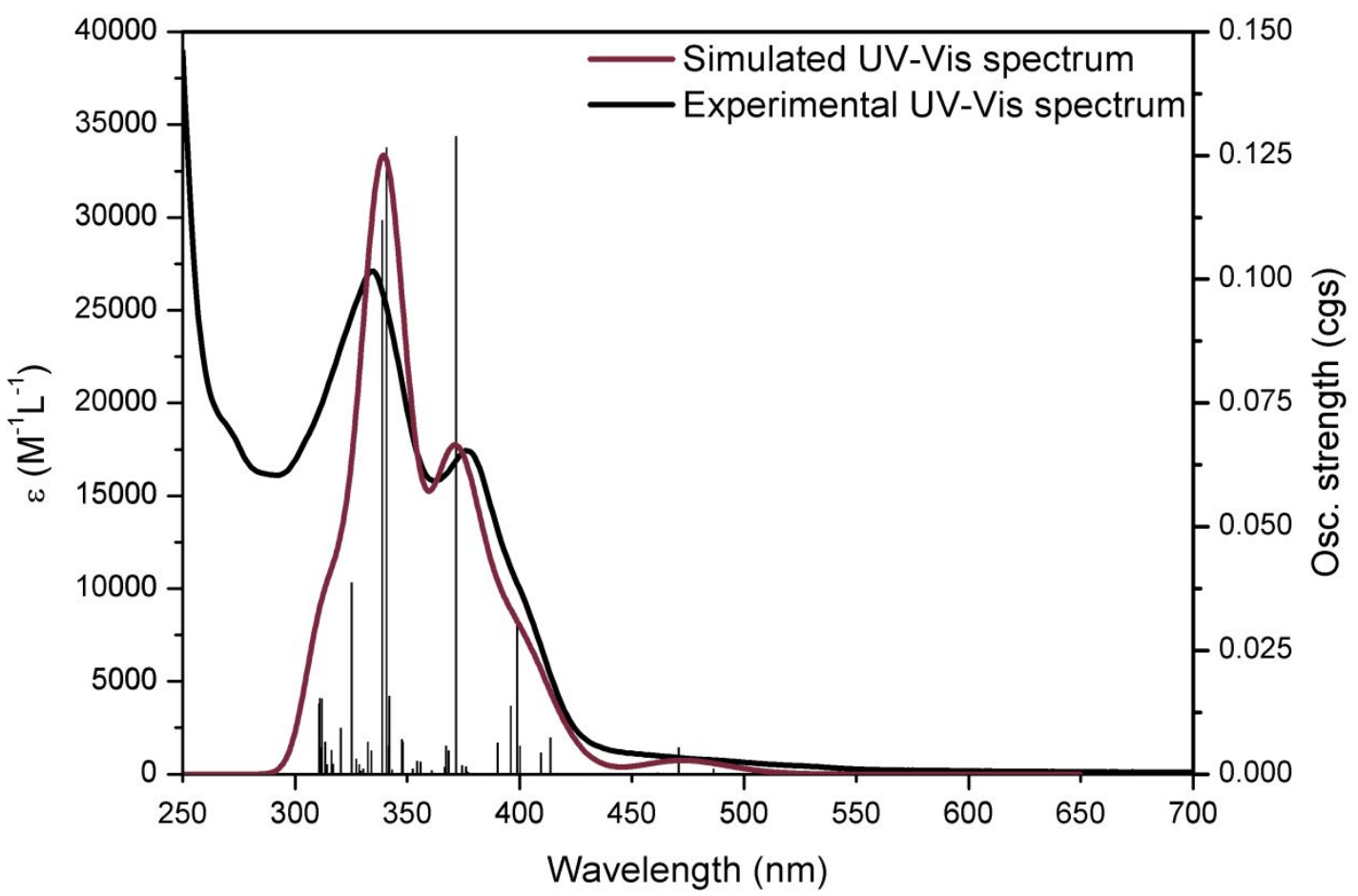

Figure S11. Experimental and simulated absorption spectrum of the complex rac$\left[\mathrm{Cr}(\mathrm{dqp})_{2}\right]^{3+}$. The vertical black lines represent the oscillatory strengths for a given calculated transition. 
Table S7. Absorption spectra recorded for $r a c-\left[\mathrm{Cr}(\mathrm{dqp})_{2}\right]\left(\mathrm{SO}_{3} \mathrm{CF}_{3}\right)_{3}$, in $\mathrm{H}_{2} \mathrm{O}$ at $2 \cdot 10^{-4} \mathrm{M}$ between $250-650 \mathrm{~nm}$ and at $7.7 \mathrm{mM}$ between $650-850 \mathrm{~nm}$ at $293 \mathrm{~K}$.

\begin{tabular}{ccccc}
\hline Compound & $\lambda(\mathrm{nm})$ & $v\left(\mathrm{~cm}^{-1}\right)$ & $\varepsilon\left(\mathrm{M}^{-1} \mathrm{~cm}^{-1}\right)$ & Assignement ${ }^{a}$ \\
\hline rac- $\left[\mathrm{Cr}(\mathrm{dqp})_{2}\right]^{3+}$ & 334 & 29940 & 37201 & $\pi^{*} \leftarrow \pi$ \\
376 & 26595 & 24094 & $\pi^{*} \leftarrow \pi$ \\
401 & 24875 & $b$ & ${ }^{4} \mathrm{~T}_{2} \leftarrow{ }^{4} \mathrm{~A}_{2}$ \\
457 & 21881 & 1424 & $\mathrm{LMCT} /(\mathrm{MC})$ \\
490 & 20408 & 967 & $\mathrm{LMCT}^{2}(\mathrm{MC})$ \\
532 & 18796 & 560 & $\mathrm{LMCT}^{2}(\mathrm{MC})$ \\
698 & 14310 & 0.1 & ${ }^{2} \mathrm{~T}_{1} \leftarrow{ }^{4} \mathrm{~A}_{2}$ \\
726 & 13770 & 0.58 & ${ }^{2} \mathrm{~T}_{1} \leftarrow{ }^{4} \mathrm{~A}_{2}$ \\
749 & 13340 & $0.1^{b}$ & ${ }^{2} \mathrm{E} \leftarrow{ }^{4} \mathrm{~A}_{2}$ \\
\hline
\end{tabular}

${ }^{a}$ Octahedral point groups $\left(O, O_{\mathrm{h}}\right)$ are assumed for the $\left[\mathrm{CrN}_{6}\right]$ chromophores, see main text. ${ }^{b}$ Extracted from TD-DFT calculations. 


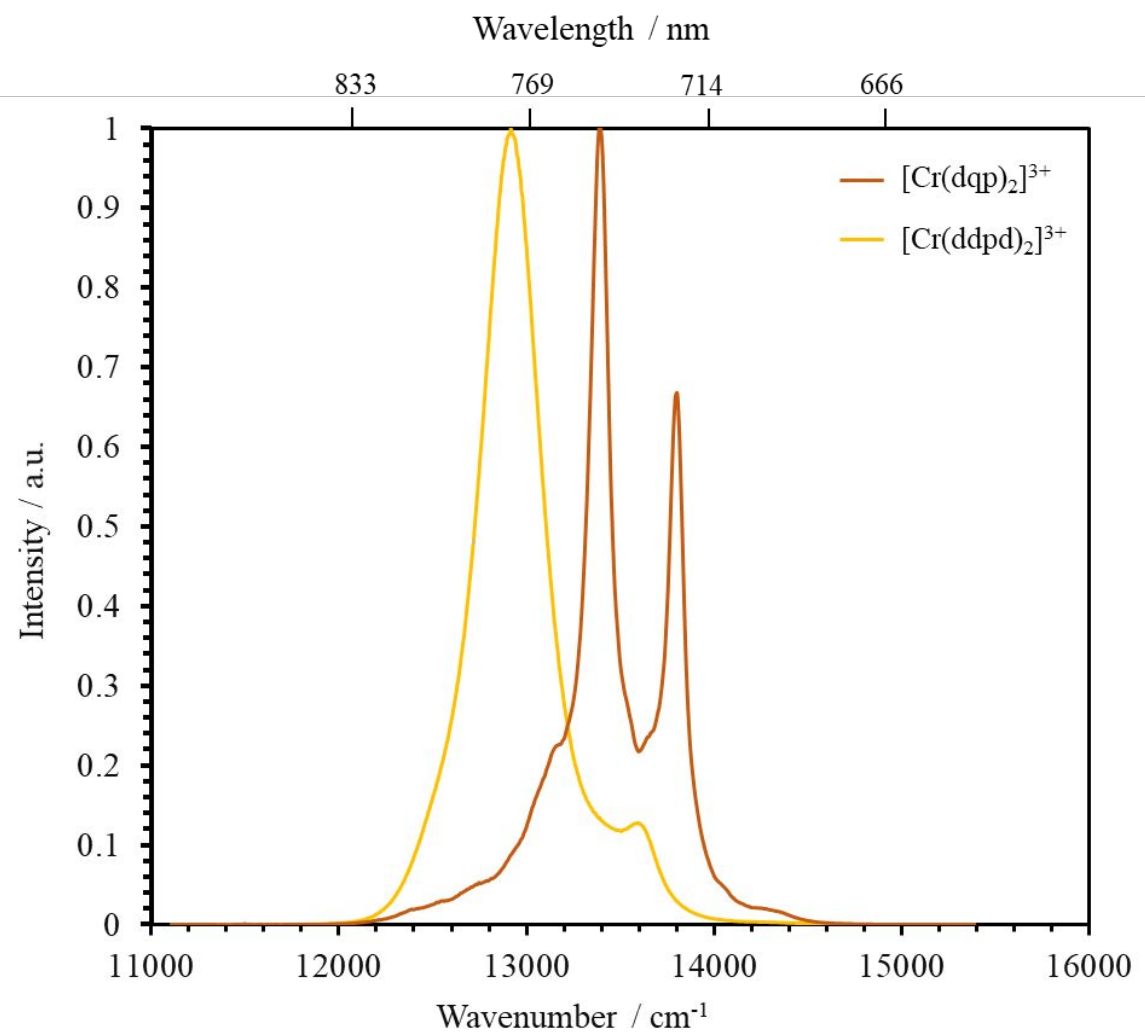

Figure S12. Emission spectrum of $r a c-\left[\mathrm{Cr}(\mathrm{dqp})_{2}\right]^{3+}$ (brown) and $\mathrm{rac}-\left[\mathrm{Cr}(\mathrm{ddpd})_{2}\right]^{3+}$ (yellow) recorded at $293 \mathrm{~K}$ at $10^{-4} \mathrm{M}$. Solvent $\mathrm{H}_{2} \mathrm{O}$. Excitation at $355 \mathrm{~nm}$.

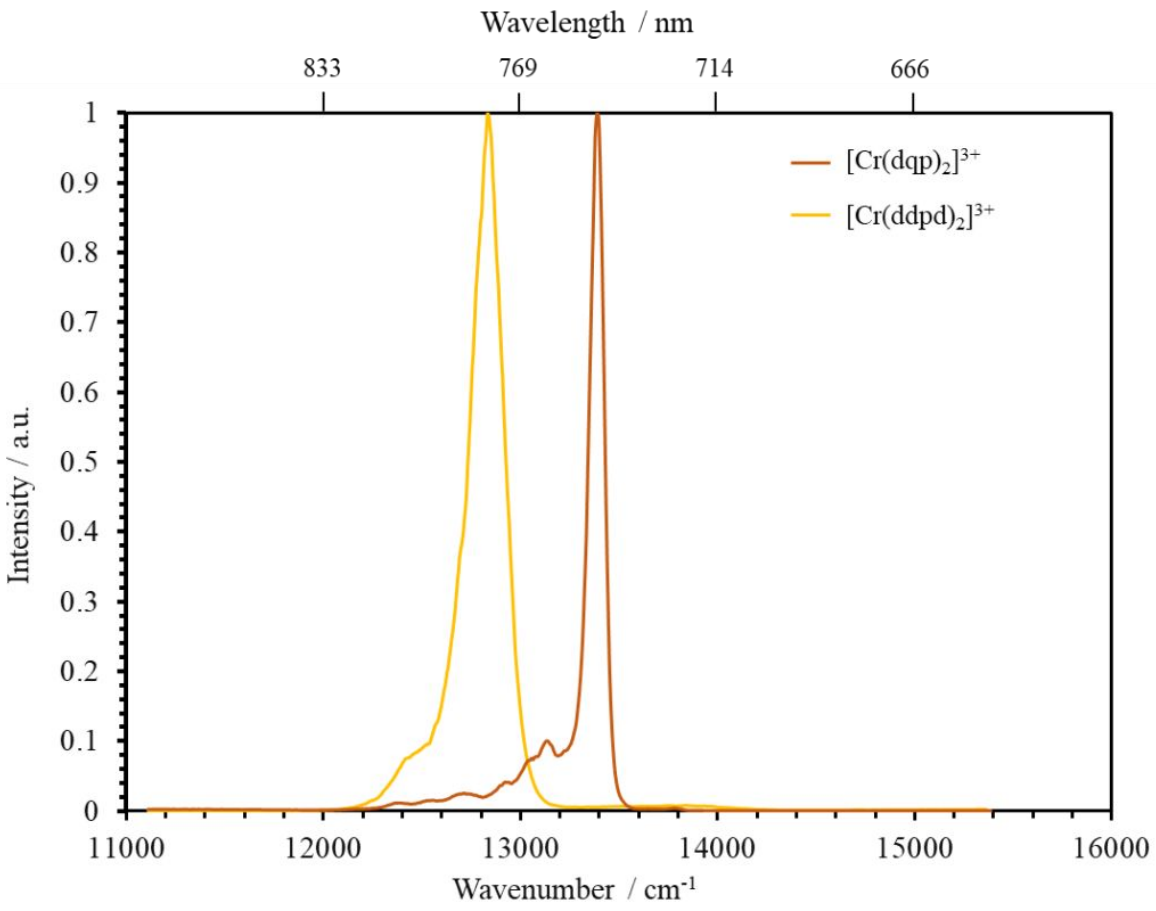

Figure S13. Emission spectrum of $r a c-\left[\mathrm{Cr}(\mathrm{dqp})_{2}\right]^{3+}$ (brown) and $\mathrm{rac}-\left[\mathrm{Cr}(\mathrm{ddpd})_{2}\right]^{3+}$ (yellow) recorded at $77 \mathrm{~K}$ at $5 \times 10^{-3} \mathrm{~mol} / \mathrm{L}$. Solvent $\mathrm{H}_{2} \mathrm{O} / \mathrm{DMSO}(5 / 5)$. Excitation at $355 \mathrm{~nm}$. 


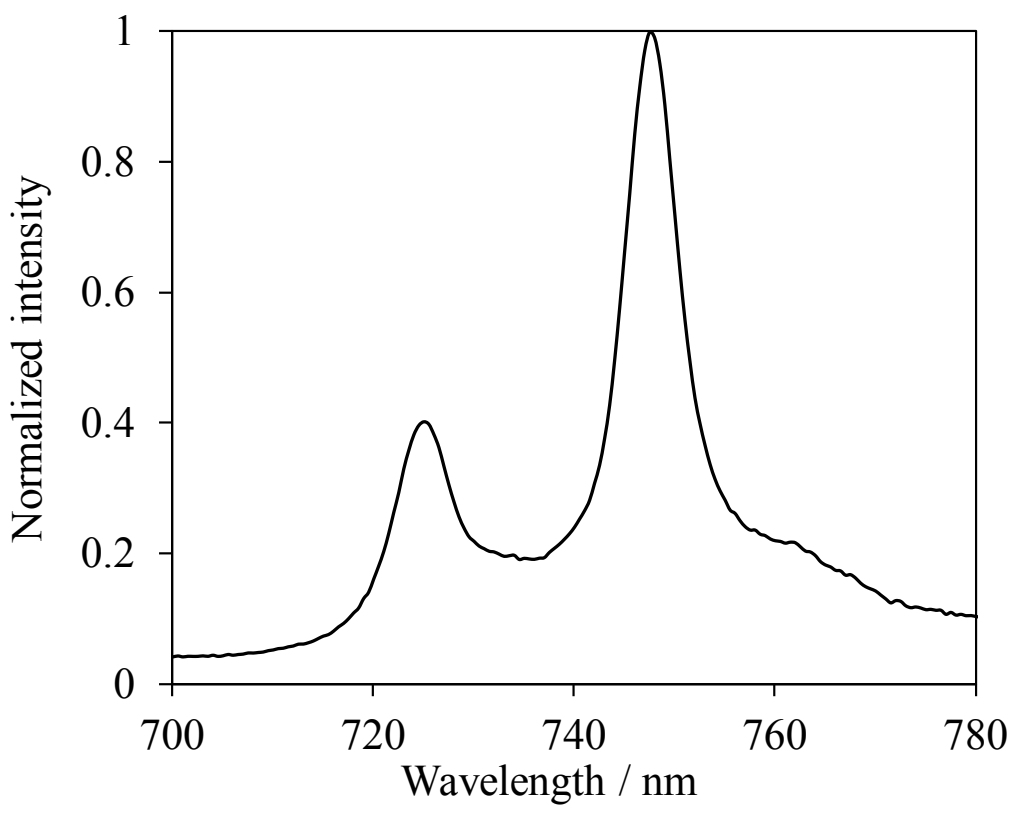

Figure S14. Emission spectrum of rac- $\left[\mathrm{Cr}(\mathrm{dqp})_{2}\right]\left(\mathrm{SO}_{3} \mathrm{CF}_{3}\right)_{3}$ recorded in solid state at $293 \mathrm{~K}$ 
Table S8. Energies of the $\operatorname{Cr}\left({ }^{4} \mathrm{~A}_{2}\right), \operatorname{Cr}\left({ }^{2} \mathrm{~T}_{1}\right)$ and $\operatorname{Cr}\left({ }^{2} \mathrm{E}\right)$ levels and ligand field $\Delta$ and Racah parameters $B$ and $C$ computed with eqs $2-4$ for $\left[\mathrm{Cr}(\mathrm{dqp})_{2}\right]^{3+}$ and $\left[\mathrm{Cr}(\mathrm{ddpd})_{2}\right]^{3+}, 54$ in solid state at $293 \mathrm{~K}$.

\begin{tabular}{lcccccccccc}
\hline Compound & $\begin{array}{c}\Delta \\
/ \mathrm{cm}^{-1}\end{array}$ & $\begin{array}{c}B \\
/ \mathrm{cm}^{-1}\end{array}$ & $\begin{array}{c}C \\
/ \mathrm{cm}^{-1}\end{array}$ & $\Delta / B$ & $C / B$ & $\begin{array}{c}{ }^{2} \mathrm{E} \\
/ \mathrm{cm}^{-1}\end{array}$ & $\begin{array}{c}{ }^{2} \mathrm{~T}_{1} \\
/ \mathrm{cm}^{-1}\end{array}$ & $\begin{array}{c}{ }^{4} \mathrm{~T}_{2} \\
/ \mathrm{cm}^{-1}\end{array}$ & $\begin{array}{c}{ }^{2} \mathrm{~T}_{2} \\
/ \mathrm{cm}^{-1 b}\end{array}$ & $\begin{array}{c}{ }^{4} \mathrm{~T}_{1} \\
/ \mathrm{cm}^{-1 b}\end{array}$ \\
\hline$\left[\mathrm{Cr}(\mathrm{dqp})_{2}\right]^{3+}$ & 24937 & 656 & 2791 & 38 & 4.3 & 13415 & 13864 & 24937 & 20758 & 32030 \\
{$\left[\mathrm{Cr}(\mathrm{ddpd})_{2}\right]^{3+}$} & 22883 & 763 & 2442 & 30 & 3.2 & 12903 & 13949 & 22883 & 19176 & 30823 \\
\hline
\end{tabular}

${ }^{a}$ The energy of $\operatorname{Cr}\left({ }^{2} \mathrm{~T}_{1}\right)$ is taken as the average of its split components (see text). ${ }^{b}$ Computed using $E\left({ }^{2} \mathrm{~T}_{2}\right)=15 B+5 C-176\left(B^{2} / \Delta\right)$ and $E\left({ }^{4} \mathrm{~T}_{1}\right)=$ $1.5 \Delta+7.5 B-0.5 \sqrt{225 B^{2}+\Delta^{2}-18 \Delta B} .56$ 


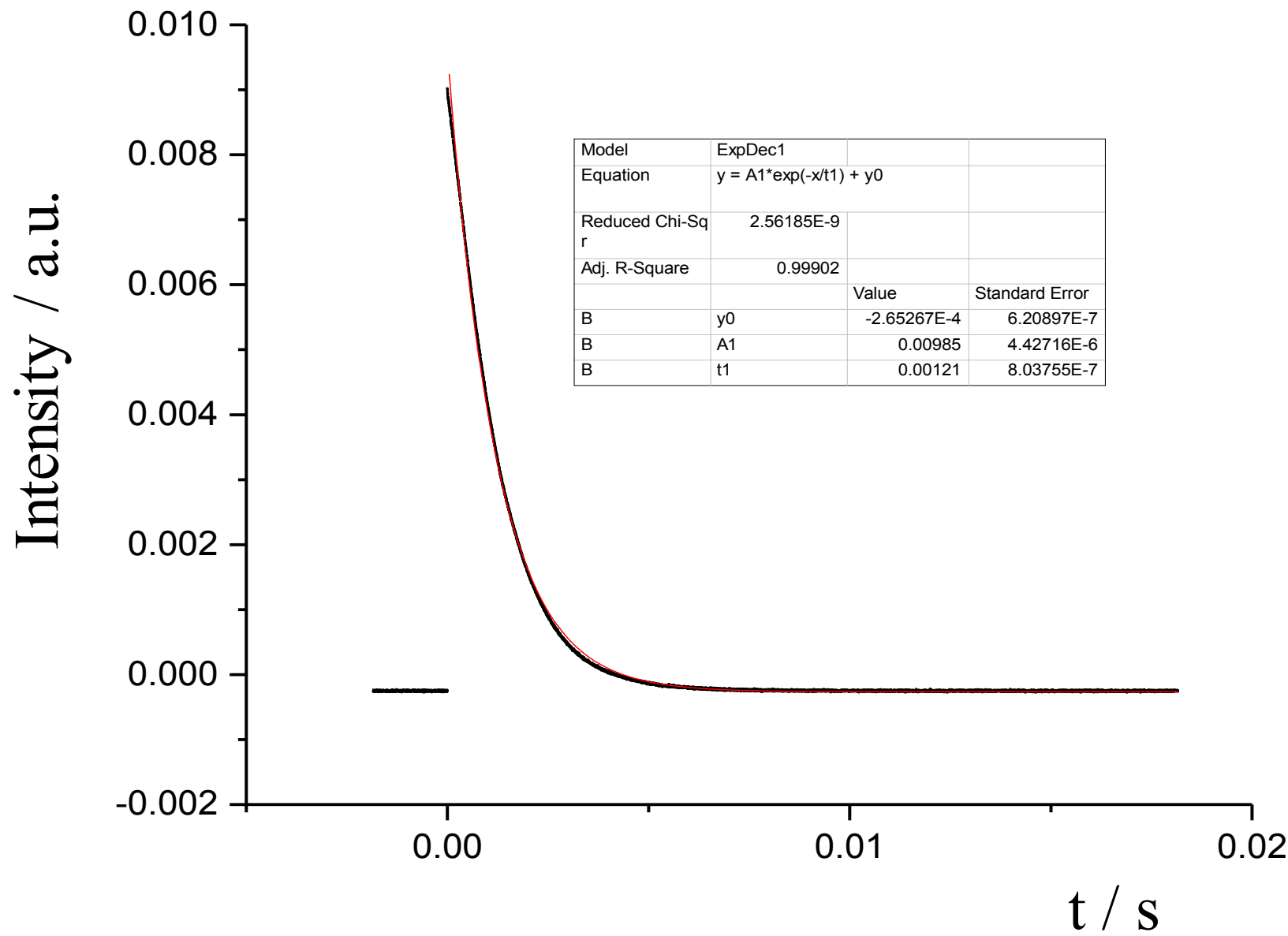

Figure S15. $\operatorname{Cr}\left({ }^{2} \mathrm{E}\right)$ emission decay curve measured in deaerated aqueous solution $\left(293 \mathrm{~K}, 10^{-4}\right.$ $\left.\mathrm{M}, \lambda_{\text {exc }}=355 \mathrm{~nm}\right)$ for $\mathrm{rac}$ - $\left[\mathrm{Cr}(\mathrm{dqp})_{2}\right]\left(\mathrm{SO}_{3} \mathrm{CF}_{3}\right)_{3}$. The black dots correspond to the experimental data and the red trace to fitted data. The fitting parameters are depicted in the associated tables. 


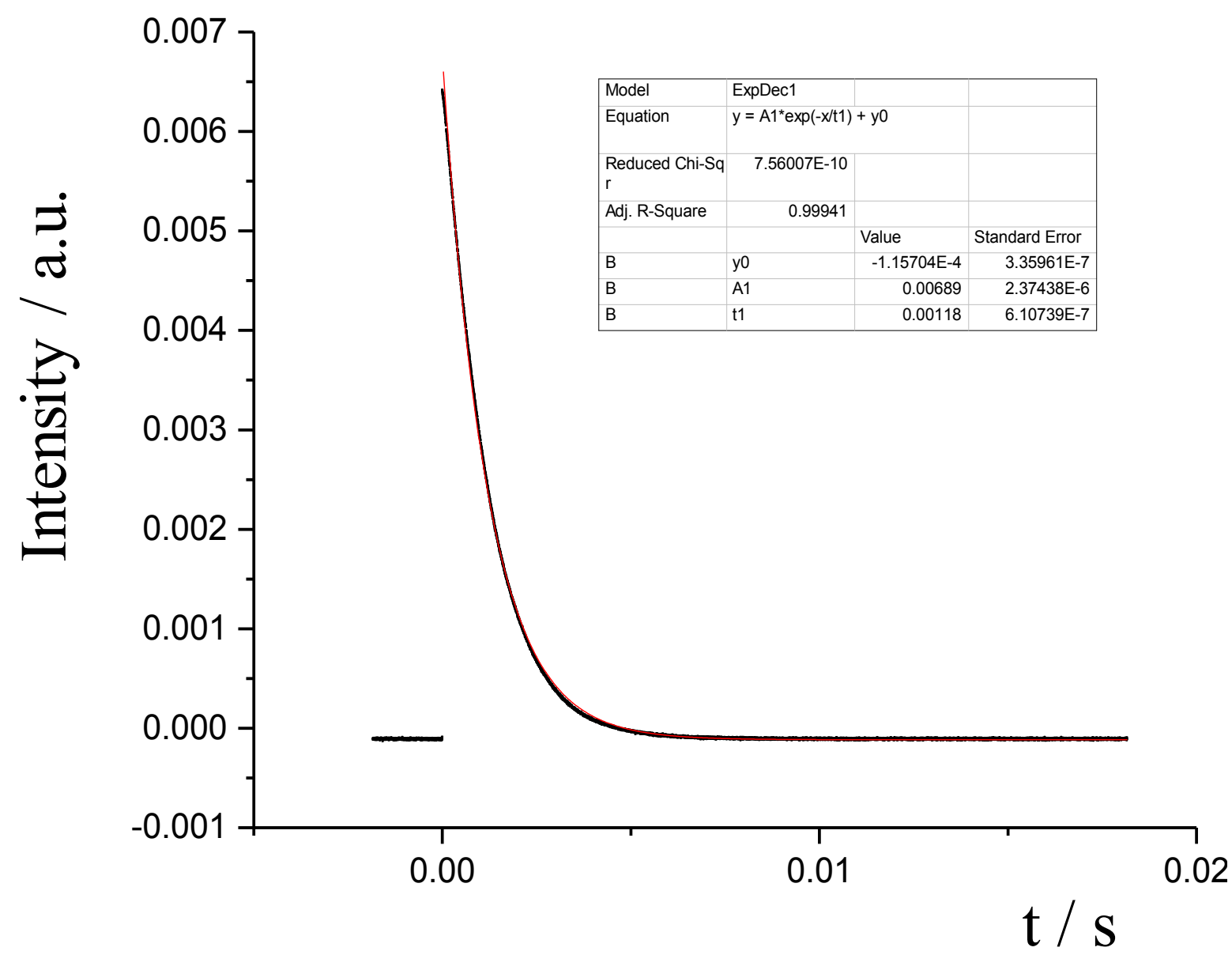

Figure S16. $\operatorname{Cr}\left({ }^{2} \mathrm{~T}_{1}\right)$ emission decay curve measured in deaerated aqueous solution $\left(293 \mathrm{~K}, 10^{-}\right.$ $\left.4 \mathrm{M}, \lambda_{\text {exc }}=355 \mathrm{~nm}\right)$ for $\mathrm{rac}-\left[\mathrm{Cr}(\mathrm{dqp})_{2}\right]\left(\mathrm{SO}_{3} \mathrm{CF}_{3}\right)_{3}$. The black dots correspond to the experimental data and the red trace to fitted data. The fitting parameters are depicted in the associated tables. 


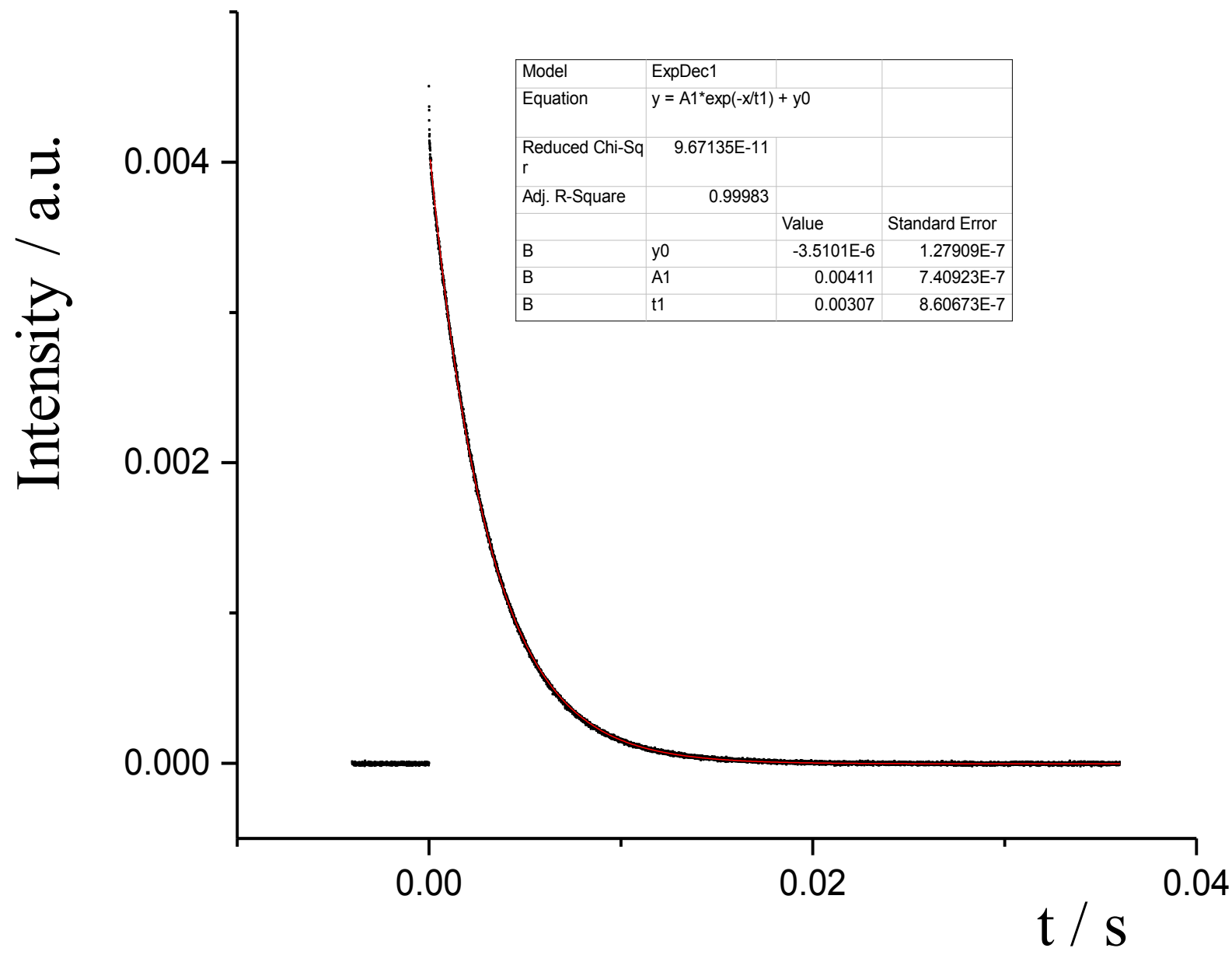

Figure S17. $\mathrm{Cr}\left({ }^{2} \mathrm{E}\right)$ emission decay curve measured in $\mathrm{H}_{2} \mathrm{O} / \mathrm{DMSO}(5 / 5)$ solution $(77 \mathrm{~K}$, at $\left.5 \times 10^{-3} \mathrm{~mol} / \mathrm{L}, \lambda_{\text {exc }}=355 \mathrm{~nm}\right)$ for $\mathrm{rac}$ - $\left[\mathrm{Cr}(\mathrm{dqp})_{2}\right]\left(\mathrm{SO}_{3} \mathrm{CF}_{3}\right)_{3}$. The black dots correspond to experimental data and the red trace to fitted data. The fitting parameter are depicted in the associated table.

Table S9. $\mathrm{Cr}\left({ }^{2} \mathrm{E}\right)$ excited-state lifetimes determined for $\left[\mathrm{Cr}(\mathrm{dqp})_{2}\right]\left(\mathrm{SO}_{3} \mathrm{CF}_{3}\right)_{3}$ in solution as well as the luminescent quantum yields, $\phi$, in aerated, deaerated solutions.

\begin{tabular}{cccccc}
\hline & $\tau_{77 \mathrm{~K}} / \mu \mathrm{s}^{a}$ & $\tau_{293 \mathrm{~K}} / \mu \mathrm{s}^{b}$ & $\tau_{293 \mathrm{~K}} / \mu \mathrm{s}^{c}$ & $\phi_{293 \mathrm{~K}}{ }^{b}$ & $\phi_{293 \mathrm{~K}}{ }^{c}$ \\
\hline$[\mathrm{Cr}(\mathrm{dqp})]^{3+}$ & 3070 & 1270 & 83 & $5.2 \%$ & $1.0 \%$ \\
\hline
\end{tabular}

${ }^{a}$ Measurement in $\mathrm{H}_{2} \mathrm{O} / \mathrm{DMSO}(5 / 5)$ solution $\left(5 \times 10^{-3} \mathrm{~mol} / \mathrm{L}\right) .{ }^{\mathrm{b}}$ Measurement in freeze-pumpthaw degassed water solution $\left(10^{-4} \mathrm{~mol} / \mathrm{L}, \lambda_{\mathrm{ex}}=410 \mathrm{~nm}\right) .{ }^{c}$ Measurements in aerated water solution $\left(10^{-4} \mathrm{~mol} / \mathrm{L}, \lambda_{\mathrm{ex}}=410 \mathrm{~nm}\right)$. 


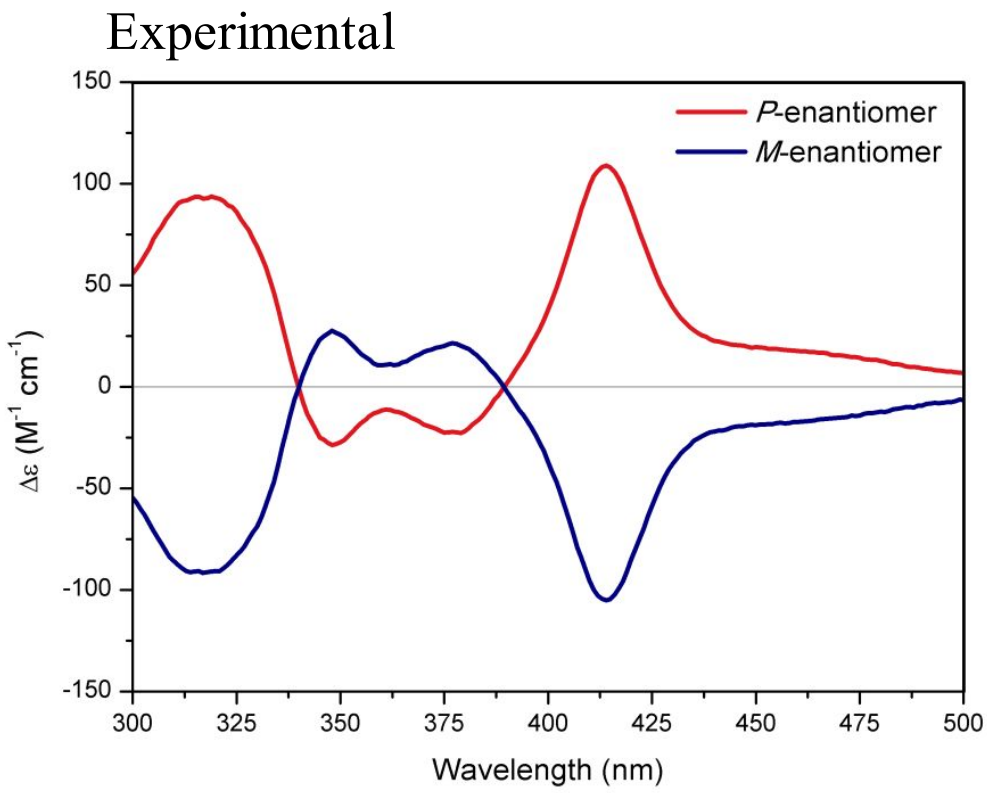

Simulated

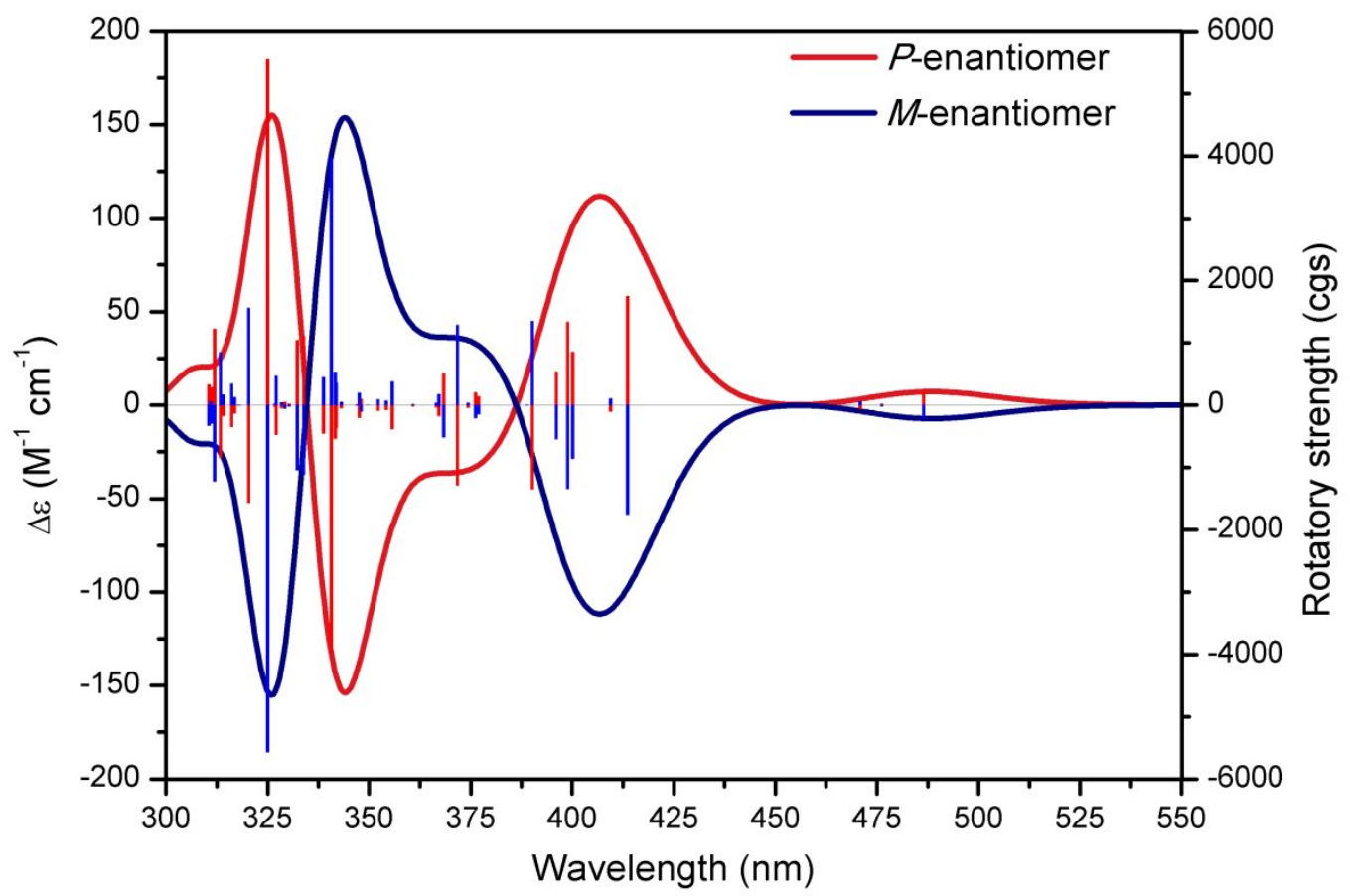

Figure S18. Experimental (top) and simulated (bottom) CD spectra of $P P-(+)-\left[\mathrm{Cr}(\mathrm{dqp})_{2}\right]^{3+}$ and $M M-(-)-\left[\mathrm{Cr}(\mathrm{dqp})_{2}\right]^{3+}$ (acetonitrile, $\left.293 \mathrm{~K}\right)$ 
Table S10. TD-DFT calculated first 30 transitions of $P P-(+)-\left[\mathrm{Cr}(\mathrm{dqp})_{2}\right]^{3+}$ and $M M-(-)-$ $\left[\mathrm{Cr}(\mathrm{dqp})_{2}\right]^{3+}(\mathrm{B} 3 \mathrm{LYP} / \mathrm{LANL} 2 \mathrm{DZ} / 6-31+\mathrm{G}$, solvent $=\mathrm{MeCN})$, assignments and corresponding Electron Density Difference Map (EDDM) at isovalue of 0.004 (purple and blue lobes indicate loss and gain of electron density respectively, hydrogens atoms are omitted for clarity). $\mathrm{LMCT}=$ Ligand Metal Charge Transfer, $\mathrm{MC}=$ Metal Centered.

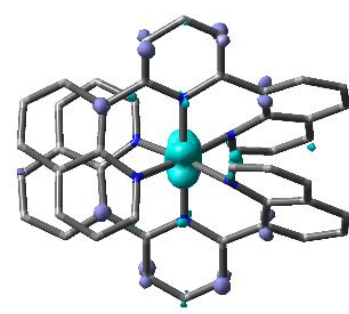

\#1

$\lambda=486.45 \mathrm{~nm}$

$\mathrm{LMCT}+{ }^{3} \pi-\pi^{*}$

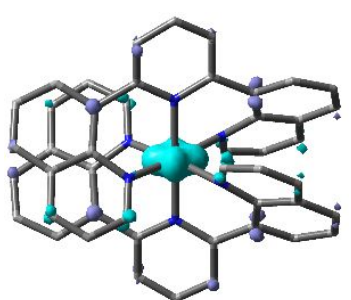

\#2

$\lambda=476.21 \mathrm{~nm}$

$\mathrm{LMCT}+{ }^{3} \pi-\pi^{*}$

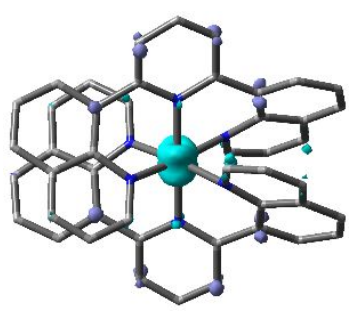

\#3

$\lambda=470.87 \mathrm{~nm}$

$\mathrm{LMCT}+{ }^{3} \pi-\pi^{*}$

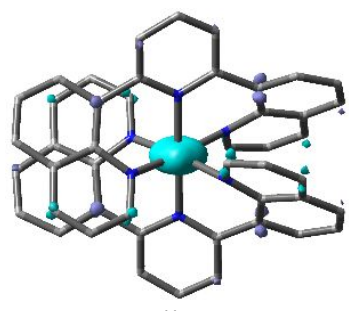

\#4

$\lambda=461.46 \mathrm{~nm}$

$\mathrm{LMCT}+{ }^{3} \pi-\pi^{*}$

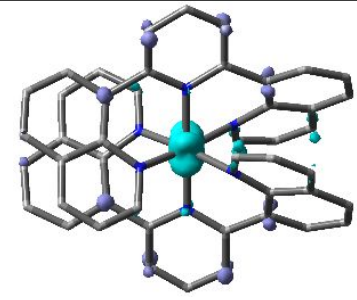

\#5

$\lambda=413.66 \mathrm{~nm}$

$\mathrm{LMCT}+\pi-\pi^{*}$

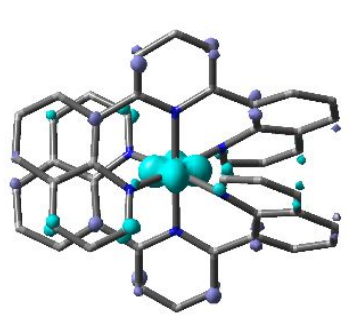

\#6

$\lambda=409.43 \mathrm{~nm}$

$\mathrm{LMCT}+\pi-\pi^{*}$

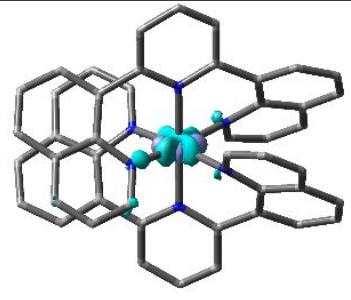

\#7

$\lambda=400.17 \mathrm{~nm}$

$\mathrm{MC}$

From: $\mathrm{t}_{2 \mathrm{~g}}\left(\mathrm{~d}_{\mathrm{xy}}\right)$

To: $e_{g}\left(d_{x-y}^{2}\right)$

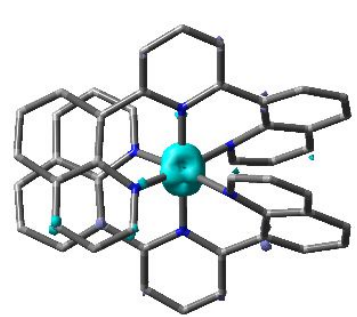

\#8

$\lambda=398.93 \mathrm{~nm}$

$\mathrm{LMCT}+\pi-\pi^{*}$

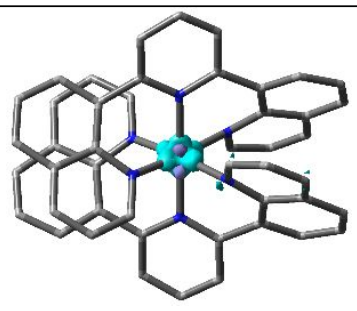

\#9

$\lambda=396.13 \mathrm{~nm}$

$\mathrm{MC}$

From: $\mathrm{t}_{2 \mathrm{~g}}\left(\mathrm{~d}_{\mathrm{xz}}\right)$

To: $e_{g}\left(d_{x-y}^{2}\right)+e_{g}$ $\left(d z^{2}\right)$

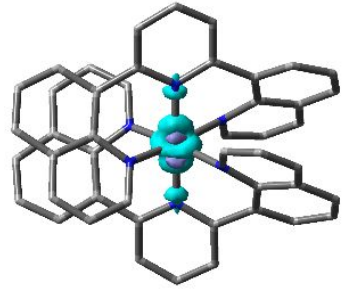

\#10

$\lambda=390.19 \mathrm{~nm}$

$\mathrm{MC}$

From: $\mathrm{t}_{2 \mathrm{~g}}\left(\mathrm{~d}_{\mathrm{xz}}\right)$

To: $\mathrm{e}_{\mathrm{g}}\left(\mathrm{d}_{\mathrm{z}}^{2}\right)$

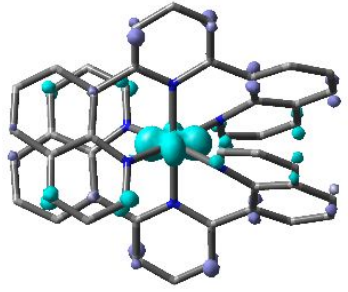

\#11

$\lambda=388.90 \mathrm{~nm}$

LMCT $+\pi-\pi^{*}$

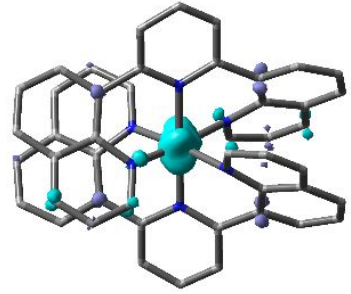

\#12

$\lambda=377.04 \mathrm{~nm}$

$\mathrm{LMCT}+\pi-\pi^{*}$ 


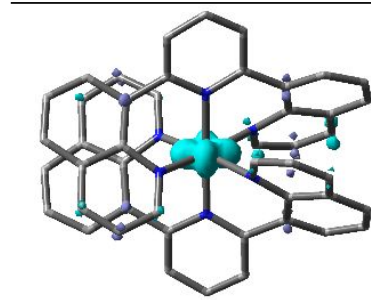

\#13

$\lambda=376.24 \mathrm{~nm}$

LMCT

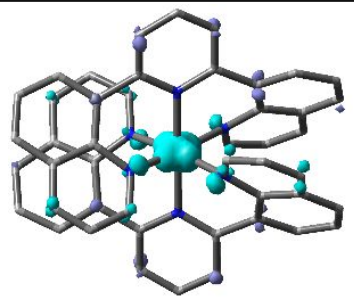

\#14

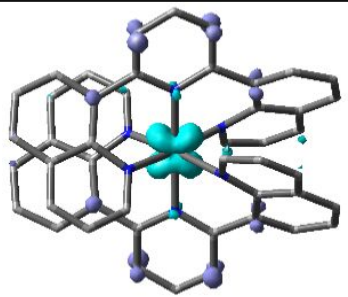

\#15

$\lambda=371.75 \mathrm{~nm}$ LMCT

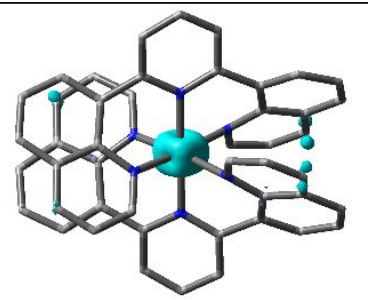

\#16

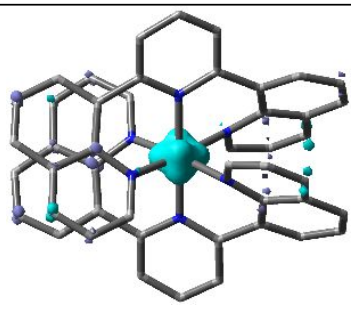

\#17

$\lambda=367.27$

LMCT

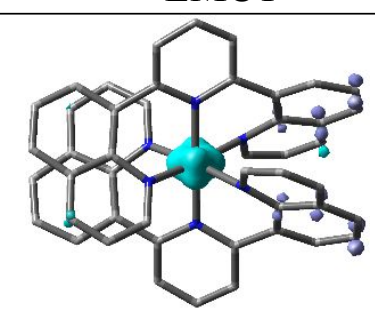

\#18

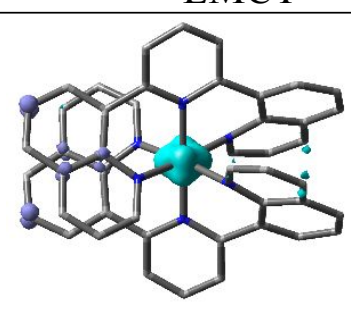

\#19

$\lambda=368.46 \mathrm{~nm}$ LMCT

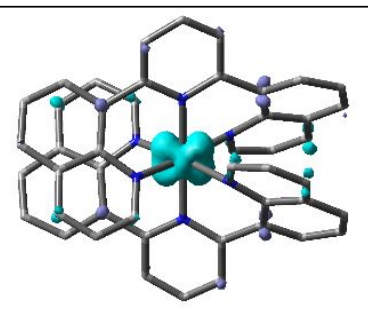

$\# 20$

$\lambda=360.85$

LMCT

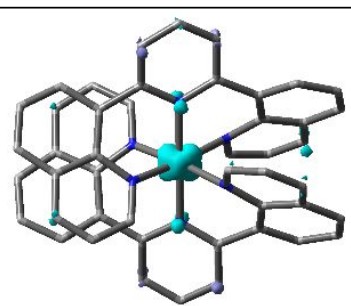

\#23

$\lambda=352.30$

LMCT

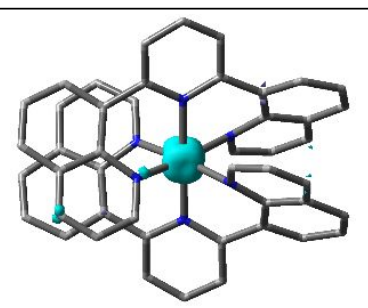

\#24

$\lambda=349.77$ LMCT

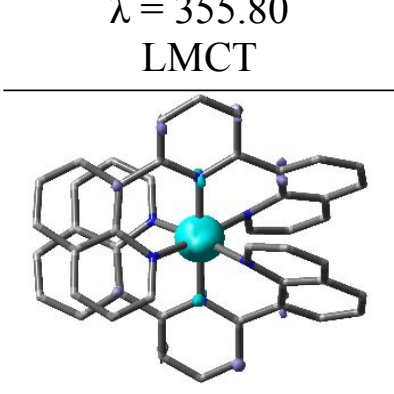

\#25

$\lambda=348.10$

LMCT

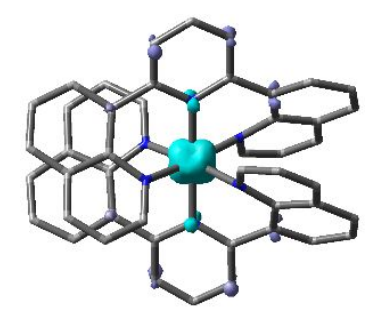

\#26

$\lambda=347.63$

LMCT

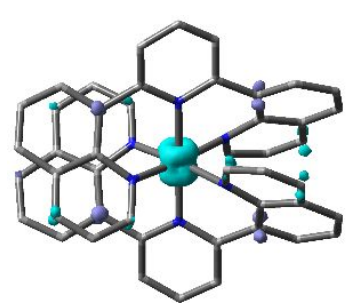

\#27

$\lambda=347.04$

LMCT

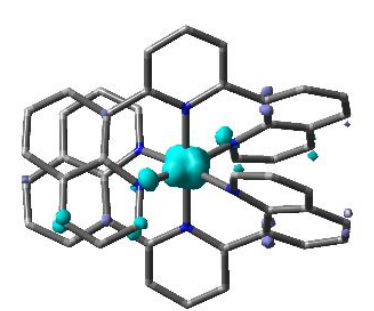

\#28

$\lambda=343.23$

LMCT 


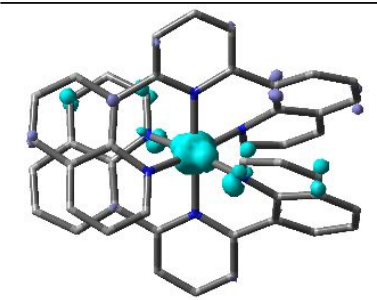

\#29

$\lambda=341.95$

LMCT

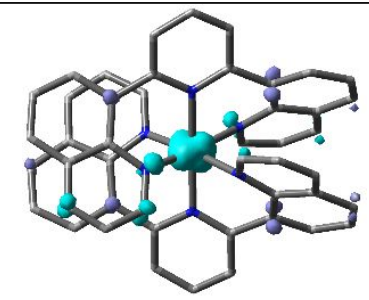

\#30

$\lambda=341.72$

$\mathrm{LMCT} / \pi-\pi^{*}$ 


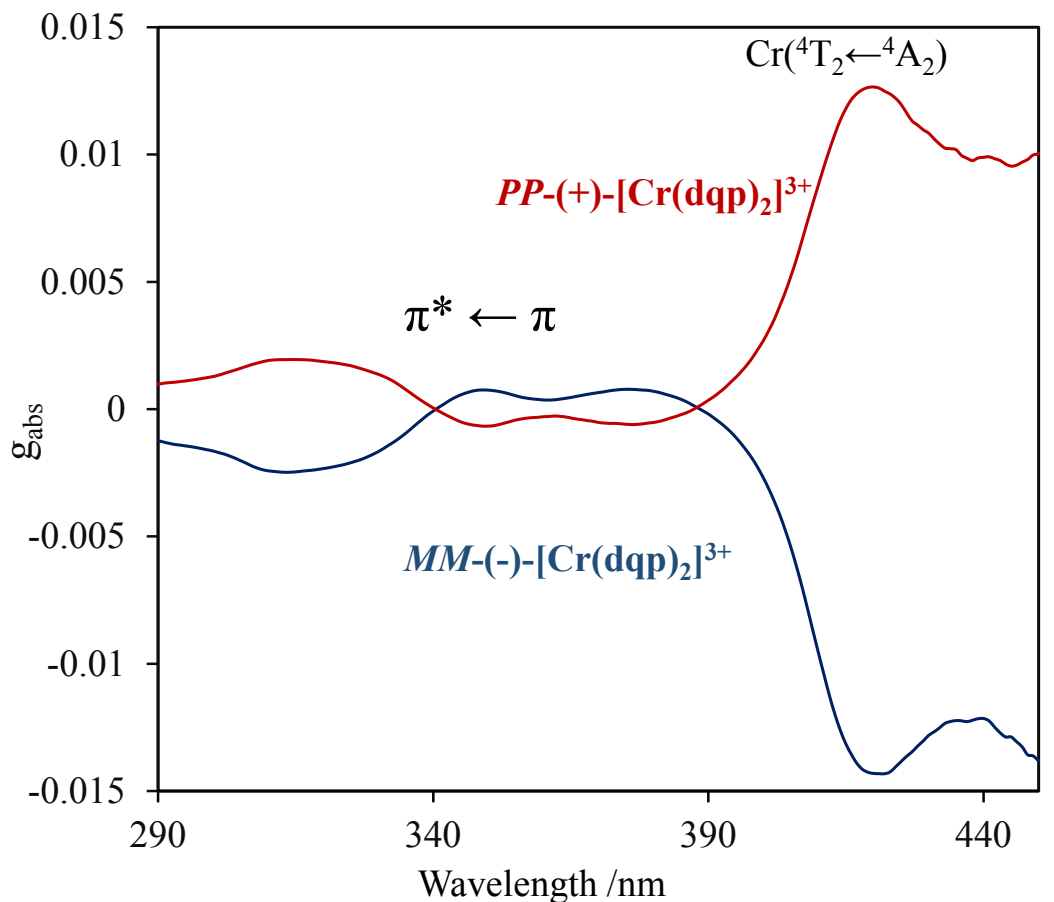

Figure S19. Absorption dissymmetry factor, $g_{\mathrm{abs}}$, for $P P-(+)-\left[\mathrm{Cr}(\mathrm{dqp})_{2}\right]^{3+}$ and $M M-(-)-$ $\left[\mathrm{Cr}(\mathrm{dqp})_{2}\right]^{3+}$ (acetonitrile, $\left.293 \mathrm{~K}\right)$.

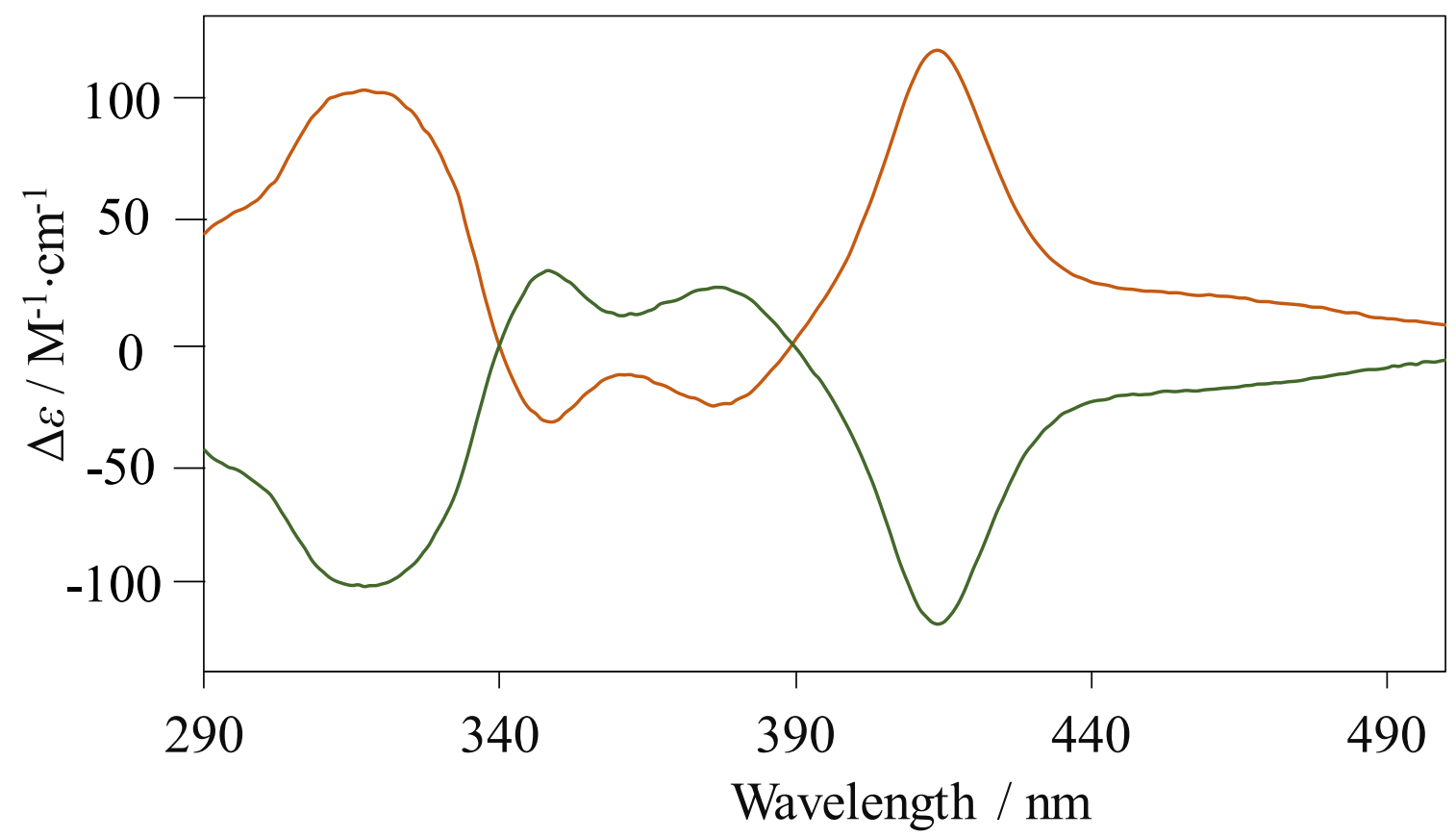

Figure S20. Experimental CD spectra of $P P-(+)-\left[\mathrm{Cr}(\mathrm{dqp})_{2}\right]^{3+}$ (brown line) and $M M-(-)-$ $\left[\mathrm{Cr}(\mathrm{dqp})_{2}\right]^{3+}$ (green line) after 45 days in an acetonitrile solution at room temperature. 


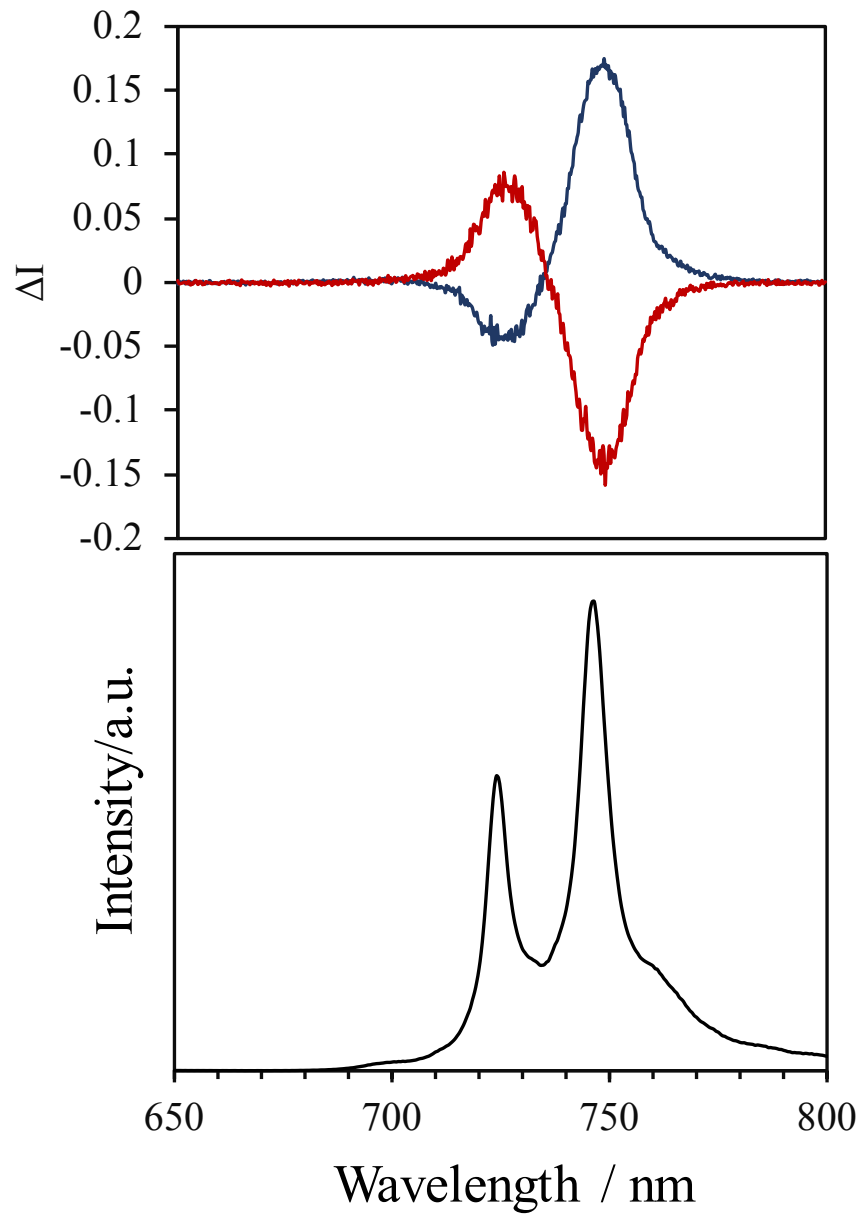

Figure S21. CPL (top) and emission (bottom) spectra recorded for $P P-(+)-\left[\mathrm{Cr}(\mathrm{dqp})_{2}\right]^{3+}$ and $M M-(-)-\left[\mathrm{Cr}(\mathrm{dqp})_{2}\right]^{3+}\left(5 \times 10^{-5} \mathrm{M}\right.$ in aerated acetonitrile, $\left.293 \mathrm{~K}, \lambda_{\mathrm{exc}}=390 \mathrm{~nm}\right)$.

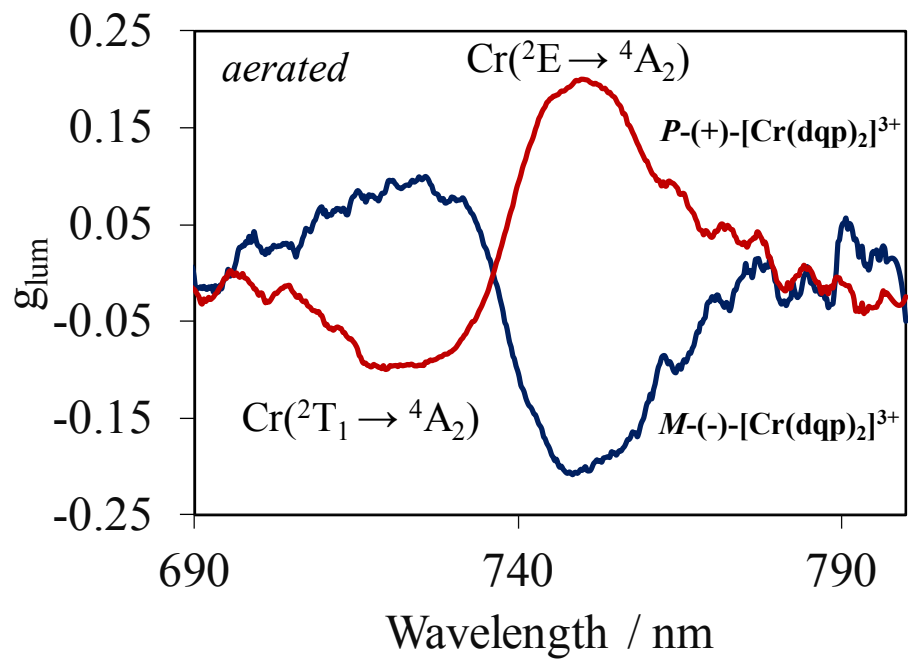

Figure S22. CPL spectra of for $P P-(+)-\left[\mathrm{Cr}(\mathrm{dqp})_{2}\right]^{3+}$ and $M M-(-)-\left[\mathrm{Cr}(\mathrm{dqp})_{2}\right]^{3+}\left(5 \times 10^{-5} \mathrm{M}\right.$ in aerated acetonitrile, $293 \mathrm{~K}, \lambda_{\mathrm{exc}}=390 \mathrm{~nm}$ ). 


\section{References}

(S1) Cantuel, M.; Bernardinelli, G.; Imbert, D.; Bünzli, J.-C. G.; Hopfgartner, G.; Piguet, C. A Kinetically Inert and Optically Active Cr(III) Partner in Thermodynamically Self-Assembled Heterodimetallic Non-Covalent d-f Podates. J. Chem. Soc. Dalt. Trans. 2002, 1929-1940.

(S2) Jäger, M.; Eriksson, L.; Bergquist, J.; Johansson, O. Synthesis and Characterization of 2,6Di(Quinolin-8-Y1)Pyridines. New Ligands for Bistridentate RuII complexes with Microsecond Luminescent Lifetimes. J. Org. Chem. 2007, 72, 10227-10230.

(S3) Yoshikawa, Y.; Yamasaki, K., Cromatographic Resolution of Metal Complexes on Sephadex Ion Exchangers. Coord. Chem. Rev. 1979, 28, 205-229

(S4) Ishida, H.; Bünzli. J.-C. G.; Beeby, A., Guidelines for Measurement of Luminescence Spectra and Quantum Yields of Inorganic and Organometallic Compounds in Solution and Solid State (IUPAC Technical Report) Pure Appl. Chem., 2016, 7, 88

(S5) Frisch, M. J.; Trucks, G. W.; Schlegel, H. B.; Scuseria, G. E.; Robb, M. A.; Cheeseman, J. R.; Scalmani, G.; Barone, V.; Mennucci, B.; Petersson, G. A.; Nakatsuji, H.; Caricato, M.; Li, X.; Hratchian, H. P.; Izmaylov, A. F.; Bloino, J.; Zheng, G.; Sonnenberg, J. L.; Hada, M.; Ehara, M.; Toyota, K.; Fukuda, R.; Hasegawa, J.; Ishida, M.; Nakajima, T.; Honda, Y.; Kitao, O.; Nakai, H.; Vreven, T.; Montgomery, J. A., Jr.; Peralta, J. E.; Ogliaro, F.; Bearpark, M.; Heyd, J. J.; Brothers, E.; Kudin, K. N.; Staroverov, V. N.; Kobayashi, R.; Normand, J.; Raghavachari, K.; Rendell, A.; Burant, J. C.; Iyengar, S. S.; Tomasi, J.; Cossi, M.; Rega, N.; Millam, J. M.; Klene, M.; Knox, J. E.; Cross, J. B.; Bakken, V.; Adamo, C.; Jaramillo, J.; Gomperts, R.; Stratmann, R. E.; Yazyev, O.; Austin, A. J.; Cammi, R.; Pomelli, C.; Ochterski, J. W.; Martin, R. L.; Morokuma, K.; Zakrzewski, V. G.; Voth, G. A.; Salvador, P.; Dannenberg, J. J.; Dapprich, S.; Daniels, A. D.; Farkas, Ö.; Foresman, J. B.; Ortiz, J. V.; Cioslowski, J.; Fox, D. J., Gaussian 09, Revision B.01, Gaussian, Inc., Wallingford CT, 2009.

(S6) Sheldrick, G. M., SHELXT - Integrated Space-group and Crystal-structure Determination. Acta Cryst. A 2015, 71, 3-8.

(S7) Sheldrick, G. M., Crystal Structure Refinement with SHELXL. Acta Cryst. C, 2015, 71, 3-8.

(S8) Dolomanov, O. V.; Bourhis, L. J.; Gildea, R. J.; Howard J. A. K.; Puschmann, H., OLEX2: a Complete Structure Solution, Refinement and Analysis Program. J Appl. Cryst. 2009, 42, $339-341$. 